T. Watanabe

Nagoya Math. J.

Vol. 170 (2003), 185-211

\title{
THE HARDY-LITTLEWOOD PROPERTY OF FLAG VARIETIES
}

\author{
TAKAO WATANABE
}

\begin{abstract}
We study the asymptotic distribution of rational points on a generalized flag variety which are of bounded height and satisfy some congruence conditions in the formulation analogous to a strongly Hardy-Littlewood variety.
\end{abstract}

Let $X$ be an affine variety in an affine space $V$ over $\mathbb{Q}$ and $B_{T}$ the set of $x \in X(\mathbb{R})$ with $\|x\| \leq T$ for a Euclidean norm $\|\cdot\|$ on $V(\mathbb{R})$. The HardyLittlewood method allows us to expect that the cardinality of $B_{T} \cap X(\mathbb{Z})$ is asymptotically equal to the volume of $B_{T}$ with respect to some measure on $X(\mathbb{R})$. On the basis of such expectation, Borovoi and Rudnick [BR] introduced the notion of a Hardy-Littlewood variety in the adelic manner. Namely, an affine variety $X$ is called a strongly Hardy-Littlewood variety if the asymptotic behavior

$$
\left|\left(B_{T} \times B_{f}\right) \cap X(\mathbb{Q})\right| \sim \omega_{X\left(\mathbb{A}_{\mathbb{Q}}\right)}\left(B_{T} \times B_{f}\right) \quad \text { as } T \rightarrow \infty
$$

holds for any open compact subset $B_{f}$ of the finite adele $X\left(\mathbb{A}_{\mathbb{Q}, f}\right)$, where $\omega_{X\left(\mathbb{A}_{\mathbb{Q}}\right)}$ denotes the measure on $X\left(\mathbb{A}_{\mathbb{Q}}\right)$ attached to a gauge form on $X$. It is known that many affine symmetric spaces have the strongly HardyLittlewood property.

In this paper, we study the asymptotic distribution of rational points of bounded height on a generalized flag variety in the formulation analogous to a strongly Hardy-Littlewood variety. Let $k$ be an algebraic number field, $G$ a connected reductive algebraic group defined over $k, Q$ a maximal $k$ parabolic subgroup of $G$ and $X=Q \backslash G$ a generalized flag variety over $k$. The adele group $G(\mathbb{A})$ of $G$ has the unimodular subgroup $G(\mathbb{A})^{1}$ consisting of all elements $g \in G(\mathbb{A})$ that satisfy $|\chi(g)|_{\mathbb{A}}=1$ for any $k$-rational character $\chi$ of $G$. Similarly, the unimodular subgroup $Q(\mathbb{A})^{1}$ of $Q(\mathbb{A})$ is defined, see Notation below for its precise definition. The homogeneous space $Y=$ $Q(\mathbb{A})^{1} \backslash G(\mathbb{A})^{1}$ is appropriate to our purpose by the reason that the set $X(k)$

Received April 6, 2001.

2000 Mathematics Subject Classification: Primary 14G05; Secondary 11G35. 
of $k$-rational points of $X$ is naturally regarded as a subset of $Y$ and there is a unique right $G(\mathbb{A})^{1}$-invariant measure $\omega_{Y}$ on $Y$ matching with Tamagawa measures $\omega_{G(\mathbb{A})^{1}}$ and $\omega_{Q(\mathbb{A})^{1}}$ of $G(\mathbb{A})^{1}$ and $Q(\mathbb{A})^{1}$, respectively. It is observed that $Y$ is decomposed into the direct product of the infinite part $Y_{\infty}$ and the finite part $Y_{f}$, and $Y_{f}$ is naturally identified with the homogeneous space $Q\left(\mathbb{A}_{f}\right) \backslash G\left(\mathbb{A}_{f}\right)$. By a strongly $k$-rational representation $\pi$ of $G$, the variety $X$ is embedded into a projective space, and the height $H_{\pi}$ is defined on $X(k)$. Since $H_{\pi}$ is extended to a positive real valued function on $Y$, we can define the "ball" $B_{T}$ of radius $T$ as the set of $y \in Y_{\infty}$ with $H_{\pi}(y) \leq T$. Then the main theorem of this paper is stated that the asymptotic behavior

$$
\left|\left(B_{T} \times B_{f}\right) \cap X(k)\right| \sim \frac{\tau(Q)}{\tau(G)} \omega_{Y}\left(B_{T} \times B_{f}\right) \quad \text { as } T \rightarrow \infty
$$

holds for any open subset $B_{f}$ of $Y_{f}$. Here $\tau(G)$ and $\tau(Q)$ stand for the Tamagawa numbers of $G$ and $Q$, respectively. In view of the equality $\left(B_{T} \times Y_{f}\right) \cap X(k)=\left\{x \in X(k): H_{\pi}(x) \leq T\right\},(0.1)$ yields the asymptotic distribution of rational points $x \in X(k)$ which satisfy $H_{\pi}(x) \leq T$ together with congruence conditions provided by $B_{f}$. The volume $\omega_{Y}\left(B_{T} \times B_{f}\right)$ is explicitly computed in the following sense. If $K_{f}$ is a good maximal compact subgroup of the finite adele group $G\left(\mathbb{A}_{f}\right)$ and $B_{f}$ is the image of an open subgroup $D_{f} \subset K_{f}$ to $Y_{f}=Q\left(\mathbb{A}_{f}\right) \backslash G\left(\mathbb{A}_{f}\right)$, then

$$
\omega_{Y}\left(B_{T} \times B_{f}\right)=\frac{\left[D_{f}\left(K_{f} \cap Q\left(\mathbb{A}_{f}\right)\right): D_{f}\right] C_{G} d_{Q}}{\left[K_{f}: D_{f}\right] C_{Q} d_{G} e_{Q}} T^{e_{Q}[k: \mathbb{Q}] / e_{\pi}},
$$

where $d_{G}, d_{Q}$ and $e_{Q}$ are positive integers depending on $G$ and $Q, e_{\pi}$ is a positive rational numbers depending on $\pi$ and these constants are easily computed. Both $C_{G}$ and $C_{Q}$ are also positive real constants depending on $G$ and $Q$, however the determination of their explicit values is more complicated than other constants. In some particular cases, e.g., the case that $G$ splits over $k$ or $G$ is a special orthogonal group, we can describe $C_{G} / C_{Q}$ by using the special values of the Dedekind zeta function of $k$ (cf. Section 7).

Our result gives an affirmative partial answer to a question mentioned in the last paragraph of [MW2, Section 4.3]. The asymptotic formula of rational points of bounded height on any generalized flag variety was first obtained by Franke, Manin and Tschinkel [FMT]. In the case of $B_{f}=Y_{f}$, Corollary to Theorem 5 in [FMT] deduces the asymptotic behavior of the 
form $\left|\left(B_{T} \times Y_{f}\right) \cap X(k)\right| \sim c T^{e_{Q}[k: \mathbb{Q}] / e_{\pi}}$, where $c$ is a constant. However, it is not clear in $[\mathrm{FMT}]$ that the leading term $c T^{e_{Q}[k: \mathbb{Q}] / e_{\pi}}$ is described in terms of the volume of $B_{T} \times Y_{f}$. In order to explain it more precisely, we mention the difference between the method of [FMT] and that of this paper. A crucial observation in [FMT] is that the height zeta function can be identified with one of the Langlands-Eisenstein series. Then, by using the analytic properties of Langlands-Eisenstein series and a standard Tauberian argument, Franke, Manin and Tschinkel established their asymptotic formula. Thus the volume $\omega_{Y}\left(B_{T} \times Y_{f}\right)$ does not occur in [FMT]. In this paper, we investigate directly the function $F_{T}(g)=\left|\left(B_{T} \times B_{f}\right) \cap X(k) g\right| \omega_{Y}\left(B_{T} \times B_{f}\right)^{-1}$ on $G(k) \backslash G(\mathbb{A})^{1}$. By using the theory of constant terms of Eisenstein series, we will prove that the inner product $\left\langle\theta, F_{T}\right\rangle$ of any pseudo-Eisenstein series $\theta$ on $G(k) \backslash G(\mathbb{A})^{1}$ and $F_{T}$ satisfies

$$
\left\langle\theta, F_{T}\right\rangle \longrightarrow \frac{\tau(Q)}{\tau(G)}\langle\theta, 1\rangle \quad \text { as } T \rightarrow \infty .
$$

This and the argument similar to [DRS] and [MW1] lead us to

$$
F_{T}(g) \longrightarrow \frac{\tau(Q)}{\tau(G)} \quad \text { as } T \rightarrow \infty
$$

for every $g \in G(k) \backslash G(\mathbb{A})^{1}$, and hence we immediately obtain (0.1). In view of this, the expression of the main term of $\left|\left(B_{T} \times B_{f}\right) \cap X(k)\right|$ by $\omega_{Y}\left(B_{T} \times B_{f}\right)$ is a significant point of our result.

Notation. As usual, $\mathbb{Z}, \mathbb{Q}, \mathbb{R}$ and $\mathbb{C}$ denote the ring of integers, the field of rational, real and complex numbers, respectively. The group of positive real numbers is denoted by $\mathbb{R}_{+}^{\times}$.

Let $k$ be an algebraic number field of finite degree over $\mathbb{Q}, \mathfrak{O}$ the ring of integers in $k$ and $\mathfrak{V}$ the set of all places of $k$. We write $\mathfrak{V}_{\infty}$ and $\mathfrak{V}_{f}$ for the sets of all infinite places and all finite places of $k$, respectively. For $v \in \mathfrak{V}, k_{v}$ denotes the completion of $k$ at $v$. If $v$ is finite, $\mathfrak{O}_{v}$ denotes the ring of integers in $k_{v}$. We fix, once and for all, a Haar measure $\mu_{v}$ on $k_{v}$ normalized so that $\mu_{v}\left(\mathfrak{D}_{v}\right)=1$ if $v \in \mathfrak{V}_{f}, \mu_{v}([0,1])=1$ if $v$ is a real place and $\mu_{v}\left(\left\{a \in k_{v}: a \bar{a} \leq 1\right\}\right)=2 \pi$ if $v$ is an imaginary place. Then the absolute value $|\cdot|_{v}$ on $k_{v}$ is defined as $|a|_{v}=\mu_{v}(a C) / \mu_{v}(C)$, where $C$ is an arbitrary compact subset of $k_{v}$ with nonzero measure. We denote by $\mathbb{A}$ the adele ring of $k$, by $\mathbb{A}_{f}$ the finite adele ring of $k$ and by $|\cdot|_{\mathbb{A}}=\prod_{v \in \mathfrak{V}}|\cdot|_{v}$ the idele norm on the idele group $\mathbb{A}^{\times}$. 
Let $G$ be a connected affine algebraic group defined over $k$. For any $k$ algebra $R, G(R)$ stands for the set of $R$-rational points of $G$. Let $\mathbf{X}^{*}(G)$ and $\mathbf{X}_{k}^{*}(G)$ be the free $\mathbb{Z}$-modules consisting of all rational characters and all $k$ rational characters of $G$, respectively. The absolute Galois group $\operatorname{Gal}(\bar{k} / k)$ acts on $\mathbf{X}^{*}(G)$. The representation of $\operatorname{Gal}(\bar{k} / k)$ in the space $\mathbf{X}^{*}(G) \otimes_{\mathbb{Z}}$ $\mathbb{Q}$ is denoted by $\sigma_{G}$ and the corresponding Artin $L$-function is denoted by $L\left(s, \sigma_{G}\right)=\prod_{v \in \mathfrak{V}_{f}} L_{v}\left(s, \sigma_{G}\right)$. We set $\sigma_{k}(G)=\lim _{s \rightarrow 1}(s-1)^{n} L\left(s, \sigma_{G}\right)$, where $n=\operatorname{rank} \mathbf{X}_{k}^{*}(G)$. Let $\omega^{G}$ be a nonzero right invariant gauge form on $G$ defined over $k$. From $\omega^{G}$ and the fixed Haar measure $\mu_{v}$ on $k_{v}$, one can construct a right invariant Haar measure $\omega_{v}^{G}$ on $G\left(k_{v}\right)$. Then, the

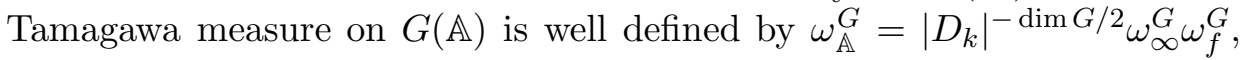
where $\omega_{\infty}^{G}=\prod_{v \in \mathfrak{V}_{\infty}} \omega_{v}^{G}, \omega_{f}^{G}=\sigma_{k}(G)^{-1} \prod_{v \in \mathfrak{V}_{f}} L_{v}\left(1, \sigma_{G}\right) \omega_{v}^{G}$ and $\left|D_{k}\right|$ is the absolute value of the discriminant of $k$. For $\chi \in \mathbf{X}_{k}^{*}(G)$, let $|\chi|_{\mathbb{A}}$ be the continuous homomorphism $G(\mathbb{A}) \rightarrow \mathbb{R}_{+}^{\times}$defined by $|\chi|_{\mathbb{A}}(g)=|\chi(g)|_{\mathbb{A}}$. We write $G(\mathbb{A})^{1}$ for the intersection of kernels of all such $|\chi|_{\mathbb{A}}$ 's. If $\chi_{1}, \ldots, \chi_{n}$ is a $\mathbb{Z}$-basis of $\mathbf{X}_{k}^{*}(G)$, then the mapping

$$
g \longmapsto\left(\left|\chi_{1}(g)\right|_{\mathbb{A}}, \ldots,\left|\chi_{n}(g)\right|_{\mathbb{A}}\right)
$$

yields an isomorphism from the quotient group $G(\mathbb{A})^{1} \backslash G(\mathbb{A})$ to $\left(\mathbb{R}_{+}^{\times}\right)^{n}$. We put the Lebesgue measure $d t$ on $\mathbb{R}$ and the invariant measure $d t / t$ on $\mathbb{R}_{+}^{\times}$. Then there exists uniquely a Haar measure $\omega_{G(\mathbb{A})^{1}}$ of $G(\mathbb{A})^{1}$ such that the Haar measure on $G(\mathbb{A})^{1} \backslash G(\mathbb{A})$ matching with $\omega_{\mathbb{A}}^{G}$ and $\omega_{G(\mathbb{A})^{1}}$ is equal to the pull-back of the measure $\prod_{i=1}^{n} d t_{i} / t_{i}$ on $\left(\mathbb{R}_{+}^{\times}\right)^{n}$ by the above isomorphism. The measure $\omega_{G(\mathbb{A})^{1}}$ is independent of the choice of a $\mathbb{Z}$-basis of $\mathbf{X}_{k}^{*}(G)$. Since $G(k)$ is a discrete subgroup of $G(\mathbb{A})^{1}$, we put the counting measure $\omega_{G(k)}$ on $G(k)$. Then the Tamagawa number $\tau(G)$ is defined to be the volume of the quotient space $G(k) \backslash G(\mathbb{A})^{1}$ with respect to the measure $\omega_{G}=$ $\omega_{G(k)} \backslash \omega_{G(\mathbb{A})^{1}}$. Here, in general, if $\mu_{A}$ and $\mu_{B}$ denote Haar measures on a locally compact unimodular group $A$ and its closed unimodular subgroup $B$, respectively, then $\mu_{B} \backslash \mu_{A}$ (resp. $\mu_{A} / \mu_{B}$ ) denotes a unique right (resp. left) $A$-invariant measure on the homogeneous space $B \backslash A$ (resp. $A / B$ ) matching with $\mu_{A}$ and $\mu_{B}$.

If $X$ is an algebraic variety defined over $k$, then $X(k)$ denotes the set of $k$-rational points of $X$. In addition, if $X$ is affine, then $X(\mathbb{A})$ and $X\left(\mathbb{A}_{f}\right)$ stands for the adele and the finite adele of $X$, respectively. We say that a subset $D$ of $X(\mathbb{A})$ is decomposable if $D$ is of the form $D_{\infty} \times D_{f}$, where $D_{\infty}$ and $D_{f}$ are subsets of $\prod_{v \in \mathfrak{V}_{\infty}} X\left(k_{v}\right)$ and $X\left(\mathbb{A}_{f}\right)$, respectively. 
If $X$ is a locally compact topological space, $C_{0}(X)$ denotes the space of all compactly supported continuous functions on $X$. If $X$ is a finite set, $|X|$ denotes the cardinal number of $X$. For two non-decreasing functions $F_{1}(T)$, $F_{2}(T)$ of real variable $T, F_{1}(T) \sim F_{2}(T)$ means $\lim _{T \rightarrow \infty} F_{1}(T) / F_{2}(T)=1$ if $F_{2}(T) \neq 0$ for $T$ large enough, otherwise, $F_{1}(T) \equiv 0$.

\section{$\S 1$. Preliminaries}

In the following, let $G$ be a connected reductive group defined over $k$. We fix a maximally $k$-split torus $S$ of $G$, a maximal $k$-torus $S_{1}$ of $G$ containing $S$, a minimal $k$-parabolic subgroup $P$ of $G$ containing $S$ and a Borel subgroup $B$ of $P$ containing $S_{1}$. Then, we denote by $\Phi_{k}$ the relative root system of $G$ with respect to $S$ and by $\Delta_{k}$ the set of simple roots of $\Phi_{k}$ corresponding to $P$.

Let $M$ be the centralizer of $S$ in $G$. Then $P$ has a Levi decomposition $P=M U$, where $U$ is the unipotent radical of $P$. For every standard $k$ parabolic subgroup $R$ of $G, R$ has a unique Levi subgroup $M_{R}$ containing $M$. We denote by $U_{R}$ the unipotent radical of $R$. Throughout this paper, we fix a maximal compact subgroup $K$ of $G(\mathbb{A})$ satisfying the following property; For every standard $k$-parabolic subgroup $R$ of $G, K \cap M_{R}(\mathbb{A})$ is a maximal compact subgroup of $M_{R}(\mathbb{A})$ and $M_{R}(\mathbb{A})$ possesses an Iwasawa decomposition $\left(M_{R}(\mathbb{A}) \cap U(\mathbb{A})\right) M(\mathbb{A})\left(K \cap M_{R}(\mathbb{A})\right)$. It is known that such maximal compact subgroup of $G(\mathbb{A})$ exists. We set $K^{R}=K \cap R(\mathbb{A}), K^{M_{R}}=$ $K \cap M_{R}(\mathbb{A}), P^{R}=M_{R} \cap P$ and $U^{R}=M_{R} \cap U$.

Let $R$ be a standard $k$-parabolic subgroup of $G$. We include the case $R=G$. Let $Z_{R}$ be the greatest central $k$-split torus in $M_{R}$. The restriction $\operatorname{map} \mathbf{X}_{k}^{*}\left(M_{R}\right) \rightarrow \mathbf{X}^{*}\left(Z_{R}\right)$ is injective. Since $\mathbf{X}_{k}^{*}\left(M_{R}\right)$ has the same rank as $\mathbf{X}^{*}\left(Z_{R}\right)$, the index

$$
d_{R}=\left[\mathbf{X}^{*}\left(Z_{R}\right): \mathbf{X}_{k}^{*}\left(M_{R}\right)\right]
$$

is finite. If $\chi_{1}, \ldots, \chi_{r}$ is a $\mathbb{Z}$-basis of $\mathbf{X}^{*}\left(Z_{R}\right)$, then the mapping $z \mapsto$ $\left(\chi_{1}(z), \ldots, \chi_{r}(z)\right)$ yields an isomorphism from $Z_{R}(\mathbb{A})$ to $\left(\mathbb{A}^{\times}\right)^{r}$. We regard $\mathbb{R}_{+}^{\times}$as a subgroup of $\mathbb{A}^{\times}$by identifying $t \in \mathbb{R}_{+}^{\times}$with the idele $t_{\mathbb{A}}=\left(t_{v}\right)$ such that $t_{v}=t$ if $v \in \mathfrak{V}_{\infty}$ and $t_{v}=1$ if $v \in \mathfrak{V}_{f}$. Let $A_{R}$ denote the inverse image of $\left(\mathbb{R}_{+}^{\times}\right)^{r}$ by the isomorphism $Z_{R}(\mathbb{A}) \rightarrow\left(\mathbb{A}^{\times}\right)^{r}$. Then $M_{R}(\mathbb{A})$ has the direct product decomposition: $M_{R}(\mathbb{A})=A_{R} M_{R}(\mathbb{A})^{1}$. The Haar measure $\mu_{A_{R}}$ on $A_{R}$ is defined to be the pull-back of the invariant measure $\prod_{i=1}^{r} d t_{i} / t_{i}$ on $\left(\mathbb{R}_{+}^{\times}\right)^{r}$ with respect to the isomorphism $z \mapsto\left(\left|\chi_{1}(z)\right|_{\mathbb{A}}, \ldots,\left|\chi_{r}(z)\right|_{\mathbb{A}}\right)$ from 
$A_{R}$ onto $\left(\mathbb{R}_{+}^{\times}\right)^{r}$. It follows from the definition of $\omega_{M_{R}(\mathbb{A})^{1}}$ that the Tamagawa measure $\omega_{\mathbb{A}}^{M_{R}}$ is decomposed into $d_{R} \mu_{A_{R}} \cdot \omega_{M_{R}(\mathbb{A})^{1}}$. Both $A_{R}$ and $\mu_{A_{R}}$ are independent of the choice of a basis of $\mathbf{X}^{*}\left(Z_{R}\right)$. We set $A_{R}^{G}=A_{R} / A_{G}$.

We define another Haar measure $\nu_{M_{R}(\mathbb{A})}$ of $M_{R}(\mathbb{A})$ as follows. Let $\omega_{\mathbb{A}}^{M}$ and $\omega_{\mathbb{A}}^{U^{R}}$ be the Tamagawa measures of $M(\mathbb{A})$ and $U^{R}(\mathbb{A})$, respectively. There is the function $\delta_{P^{R}}$ on $M(\mathbb{A})$ such that the integration formula

$$
\int_{U^{R}(\mathbb{A})} f\left(m u m^{-1}\right) d \omega_{\mathbb{A}}^{U^{R}}(u)=\delta_{P^{R}}(m)^{-1} \int_{U^{R}(\mathbb{A})} f(u) d \omega_{\mathbb{A}}^{U^{R}}(u)
$$

holds for $f \in C_{0}\left(U^{R}(\mathbb{A})\right)$. In other words, $\delta_{P^{R}}^{-1}$ is the modular character of $P^{R}(\mathbb{A})$. Let $\nu_{K^{M_{R}}}$ be the Haar measure on $K^{M_{R}}$ normalized so that the total volume equals one. Then the mapping

$$
\begin{array}{r}
f \longmapsto \int_{U^{R}(\mathbb{A}) \times M(\mathbb{A}) \times K^{M_{R}}} f(u m h) \delta_{P^{R}}(m)^{-1} d \omega_{\mathbb{A}}^{U^{R}}(u) d \omega_{\mathbb{A}}^{M}(m) d \nu_{K^{M_{R}}}(h), \\
\left(f \in C_{0}\left(M_{R}(\mathbb{A})\right)\right)
\end{array}
$$

defines an invariant measure on $M_{R}(\mathbb{A})$ and is denoted by $\nu_{M_{R}(\mathbb{A})}$. There exists a positive constant $C_{R}$ such that

$$
\omega_{\mathbb{A}}^{M_{R}}=C_{R} \nu_{M_{R}(\mathbb{A})} .
$$

We have the following compatibility formula:

$$
\begin{aligned}
& \int_{G(\mathbb{A})} f(g) d \omega_{\mathbb{A}}^{G}(g) \\
& =\frac{C_{G}}{C_{R}} \int_{U_{R}(\mathbb{A}) \times M_{R}(\mathbb{A}) \times K} f(u m h) \delta_{R}(m)^{-1} d \omega_{\mathbb{A}}^{U_{R}}(u) d \omega_{\mathbb{A}}^{M_{R}}(m) d \nu_{K}(h)
\end{aligned}
$$

for $f \in C_{0}(G(\mathbb{A}))$, where $\delta_{R}^{-1}$ is the modular character of $R(\mathbb{A})$.

On the homogeneous space $Y_{R}=R(\mathbb{A})^{1} \backslash G(\mathbb{A})^{1}$, we define the right $G(\mathbb{A})^{1}$-invariant measure $\omega_{Y_{R}}$ by $\omega_{R(\mathbb{A})^{1}} \backslash \omega_{G(\mathbb{A})^{1}}$. We note that both $G(\mathbb{A})^{1}$ and $R(\mathbb{A})^{1}$ are unimodular. We identify $Y_{R}$ with $A_{G} R(\mathbb{A})^{1} \backslash G(\mathbb{A})$. Then the mapping

$$
\iota_{R}: K / K^{R} \times A_{R}^{G} \longrightarrow Y_{R}:(\bar{h}, \bar{z}) \longmapsto A_{G} R(\mathbb{A})^{1} z^{-1} h^{-1}
$$

is a bijection, where $\bar{h}=h K^{R}$ and $\bar{z}=z A_{G}$ for $h \in K$ and $z \in A_{R}$. Set $\nu_{A_{R}^{G}}=\mu_{A_{R}} / \mu_{A_{G}}$. 
LEMmA 1. Let $D$ be an open subgroup of $K$ and $\left\{h_{1}, \ldots, h_{s}\right\}$ be a complete set of coset representatives of $K / D$. Then, for any right D-invariant function $f \in C_{0}\left(Y_{R}\right)$, one has

$$
\int_{Y_{R}} f(y) d \omega_{Y_{R}}(y)=\frac{C_{G} d_{R}}{[K: D] C_{R} d_{G}} \sum_{i=1}^{s} \int_{A_{R}^{G}} f\left(\iota_{R}\left(\bar{h}_{i}^{-1}, \bar{z}\right)\right) \delta_{R}(z) d \nu_{A_{R}^{G}}(\bar{z}) .
$$

Proof. If we set

$$
\varphi(y)=\int_{K} f(y h) d \nu_{K}(h)=\frac{1}{[K: D]} \sum_{i=1}^{s} f\left(y h_{i}\right)
$$

then $\varphi$ is a right $K$-invariant function on $Y_{R}$. By [W, Corollary to Lemma 1],

$$
\int_{Y_{R}} \varphi(y) d \omega_{Y_{R}}(y)=\frac{C_{G} d_{R}}{C_{R} d_{G}} \int_{A_{R}^{G}} \varphi\left(\iota_{R}(\bar{e}, \bar{z})\right) \delta_{R}(z) d \nu_{A_{R}^{G}}(\bar{z}) .
$$

Since $\omega_{Y_{R}}$ is right $G(\mathbb{A})^{1}$-invariant, the left hand side equals the integral of $f(y)$ over $Y_{R}$.

\section{$\S 2$. Heights on flag varieties}

Let $V_{\pi}$ be a finite dimensional $\bar{k}$-vector space endowed with a $k$ structure $V_{\pi}(k)$ and $\pi: G \rightarrow G L\left(V_{\pi}\right)$ be an absolutely irreducible $k$-rational representation. The highest weight space in $V_{\pi}$ with respect to $B$ is denoted by $x_{\pi}$. Let $Q_{\pi}$ be the stabilizer of $x_{\pi}$ in $G$ and $\lambda_{\pi}$ the $\bar{k}$-rational character of $Q_{\pi}$ by which $Q_{\pi}$ acts on $x_{\pi}$. The representation $\pi$ is said to be strongly $k$-rational if $x_{\pi}$ is defined over $k$. Then $Q_{\pi}$ is a standard $k$-parabolic subgroup of $G$ and $\lambda_{\pi}$ is a $k$-rational character of $Q_{\pi}$. It is known that $\left.\lambda_{\pi}\right|_{S}$ is a non-negative integral linear combination of the fundamental $k$-weights ([W, Section 1]). We say $\pi$ is maximal if $Q_{\pi}$ is a standard maximal $k$-parabolic subgroup. This is equivalent to the condition that $\left.\lambda_{\pi}\right|_{S}$ is a positive integer multiple of a single fundamental $k$-weight.

Let $\pi$ be a strongly $k$-rational representation. For convenience, we use a right action of $G$ on $V_{\pi}$ defined by $a \cdot g=\pi\left(g^{-1}\right) a$ for $g \in G$ and $a \in V_{\pi}$. Then the mapping $g \mapsto x_{\pi} \cdot g$ gives rise to a $k$-rational embedding of $Q_{\pi} \backslash G$ into the projective space $\mathbb{P} V_{\pi}$.

We write $X_{Q_{\pi}}$ for $Q_{\pi} \backslash G$. Since $Q_{\pi}$ is a $k$-parabolic subgroup, $X_{Q_{\pi}}(k)$ is naturally identified with $Q_{\pi}(k) \backslash G(k)$ ([B, Proposition 20.5]). Let us define 
a height on $X_{Q_{\pi}}(k)$. We fix a $k$-basis $\mathbf{e}_{1}, \ldots, \mathbf{e}_{n}$ of the $k$-vector space $V_{\pi}(k)$ and define a local height $H_{v}$ on $V_{\pi}\left(k_{v}\right)$ for each $v \in \mathfrak{V}$ as follows:

$H_{v}\left(a_{1} \mathbf{e}_{1}+\cdots+a_{n} \mathbf{e}_{n}\right)= \begin{cases}\left(\left|a_{1}\right|_{v}^{2}+\cdots+\left|a_{n}\right|_{v}^{2}\right)^{1 /(2[k: \mathbb{Q}])} & (\text { if } v \text { is real }) \\ \left(\left|a_{1}\right|_{v}+\cdots+\left|a_{n}\right|_{v}\right)^{1 /[k: \mathbb{Q}]} & (\text { if } v \text { is imaginary) } \\ \sup \left(\left|a_{1}\right|_{v}, \cdots,\left|a_{n}\right|_{v}\right)^{1 /[k: \mathbb{Q}]} & \left(\text { if } v \in \mathfrak{V}_{f}\right)\end{cases}$

The global height $H_{\pi}$ on $V_{\pi}(k)$ is defined to be the product of all $H_{v}$, that is, $H_{\pi}(a)=\prod_{v \in \mathfrak{V}} H_{v}(a)$. By the product formula, $H_{\pi}$ is invariant by scalar multiplications. Thus, $H_{\pi}$ defines a height on $\mathbb{P} V_{\pi}(k)$, and on $X_{Q_{\pi}}(k)$ by restriction. The height $H_{\pi}$ is extended to $G L\left(V_{\pi}, \mathbb{A}\right) \mathbb{P} V_{\pi}(k)$ by

$$
H_{\pi}(\xi \bar{a})=\prod_{v \in \mathfrak{V}} H_{v}\left(\xi_{v} a\right)
$$

for $\xi=\left(\xi_{v}\right) \in G L\left(V_{\pi}, \mathbb{A}\right)$ and $\bar{a}=k a \in \mathbb{P} V_{\pi}(k), a \in V_{\pi}(k)-\{0\}$. We set

$$
\Phi_{\pi, \xi}(g)=H_{\pi}\left(\xi\left(x_{\pi} \cdot g\right)\right) / H_{\pi}\left(\xi x_{\pi}\right)
$$

for $g \in G(\mathbb{A})$. Obviously, $\Phi_{\pi, \xi}$ is a continuous function on $G(\mathbb{A})$ and satisfies

$$
\Phi_{\pi, \xi}(q g)=\left|\lambda_{\pi}(q)^{-1}\right|_{\mathbb{A}}^{1 /[k: \mathbb{Q}]} \Phi_{\pi, \xi}(g)
$$

for any $q \in Q_{\pi}(\mathbb{A})$ and $g \in G(\mathbb{A})$. Thus $\Phi_{\pi, \xi}$ defines a function on $Y_{Q_{\pi}}=Q_{\pi}(\mathbb{A})^{1} \backslash G(\mathbb{A})^{1}$. It is always possible that one choose an element $\xi \in G L\left(V_{\pi}, \mathbb{A}\right)$ so that $\Phi_{\pi, \xi}$ is right $K$-invariant. In many examples, one can take the identity as such $\xi$.

\section{$\S 3$. The Hardy-Littlewood property of flag varieties}

In the following, we assume $\pi$ is maximal and strongly $k$-rational. We fix, once and for all, an element $\xi \in G L\left(V_{\pi}, \mathbb{A}\right)$ such that $\Phi_{\pi, \xi}$ is right $K$ invariant. We simply write $Q$ for $Q_{\pi}$ and $\Phi_{\pi}$ for $\Phi_{\pi, \xi}$. Let $\Delta_{Q}$ be the set of nonzero roots $\left.\beta\right|_{Z_{Q}}, \beta \in \Delta_{k}$. Since $Q$ is maximal, $\Delta_{Q}$ consists of a single element $\left.\alpha\right|_{Z_{Q}}$. Let $n_{Q}$ be the positive integer such that $\left.n_{Q}^{-1} \alpha\right|_{Z_{Q}}$ is a $\mathbb{Z}$-base of $\mathbf{X}^{*}\left(Z_{G} \backslash Z_{Q}\right)$. We set $\alpha_{Q}=\left.n_{Q}^{-1} \alpha\right|_{Z_{Q}}$. Then the Haar measure $\nu_{A_{Q}}$ equals the pull-back of the measure $d t / t$ by the isomorphism $\left|\alpha_{Q}\right|_{\mathbb{A}}: A_{Q}^{G} \rightarrow \mathbb{R}_{+}^{\times}$. If we set $e_{Q}=n_{Q} \operatorname{dim} U_{Q}$, we have

$$
\delta_{Q}(z)=\left|\alpha_{Q}(z)\right|_{\mathbb{A}}^{e_{Q}}, \quad\left(z \in Z_{Q}(\mathbb{A})\right) .
$$


The quotient morphism $Z_{Q} \rightarrow Z_{G} \backslash Z_{Q}$ induces an isomorphism $\mathbf{X}^{*}\left(Z_{G} \backslash Z_{Q}\right)$ $\otimes_{\mathbb{Z}} \mathbb{Q} \rightarrow \mathbf{X}^{*}\left(Z_{Q} \cap G^{s s}\right) \otimes_{\mathbb{Z}} \mathbb{Q}$, where $G^{s s}$ denotes the derived group of $G$. Under the identification $\mathbf{X}^{*}\left(Z_{Q} \cap G^{s s}\right) \otimes_{\mathbb{Z}} \mathbb{Q} \cong \mathbf{X}^{*}\left(Z_{G} \backslash Z_{Q}\right) \otimes_{\mathbb{Z}} \mathbb{Q}$, there exists the positive rational number $e_{\pi}$ such that

$$
\left.\lambda_{\pi}\right|_{Z_{Q} \cap G^{s s}}=e_{\pi} \alpha_{Q} .
$$

Then $\Phi_{\pi}\left(\iota_{Q}(\bar{h}, \bar{z})\right)=\left|\alpha_{Q}(z)\right|_{\mathbb{A}}^{e_{\pi} /[k: \mathbb{Q}]}$ holds for any $(\bar{h}, \bar{z}) \in K / K^{Q} \times A_{Q}^{G}$.

For an open subset $D$ of $K$ and $0<T$, we set

$$
E_{\pi}(D, T)=\left\{\iota_{Q}(\bar{h}, \bar{z}): \bar{h} \in D K^{Q} / K^{Q}, \bar{z} \in A_{Q}^{G},\left|\alpha_{Q}(\bar{z})\right|_{\mathbb{A}} \leq T^{[k: \mathbb{Q}] / e_{\pi}}\right\} .
$$

Obviously, $E_{\pi}(D, T)$ is contained in $\left\{y \in Y_{Q}: \Phi_{\pi}(y) \leq T\right\}$, and in particular, the set $E_{\pi}(K, T) \cap X_{Q}(k)$ coincides with the set $\left\{x \in X_{Q}(k): H_{\pi}(\xi x) \leq\right.$ $\left.H_{\pi}\left(\xi x_{\pi}\right) T\right\}$. The next is the main theorem of this paper.

Theorem 1. Let $\pi$ and $Q$ be as above and $D=D_{\infty} \times D_{f}$ a decomposable open subset of $K$ such that $D_{\infty}$ equals the infinite part $K_{\infty}$ of $K$. Then one has

$$
\left|E_{\pi}(D, T) \cap X_{Q}(k) g\right| \sim \frac{\tau(Q)}{\tau(G)} \omega_{Y_{Q}}\left(E_{\pi}(D, T)\right) \quad \text { as } T \rightarrow \infty
$$

for any $g \in G(\mathbb{A})^{1}$.

We fix a decomposable open subset $D$ of $K$ with $D_{\infty}=K_{\infty}$. Since the finite part of $K$ is totally disconnected, there is a decomposable open normal subgroup $D_{1}$ of $K$ and $b_{0} \in D$ such that $D_{1} b_{0}^{-1} D=b_{0}^{-1} D$ and $D_{1, \infty}=K_{\infty}$. If $b_{1}, \ldots, b_{s} \in D$ is a complete set of coset representatives of $D_{1} K^{Q} \backslash b_{0}^{-1} D K^{Q}$, then $E_{\pi}\left(b_{0}^{-1} D, T\right)=E_{\pi}(D, T) b_{0}$ decomposes into a disjoint union of $E_{\pi}\left(D_{1}, T\right) b_{i}, i=1,2, \ldots, s$. It is easy to see that the truth of (3.3) for $D_{1}$ implies the truth of (3.3) for $D$. Hence, we may assume without loss of generality that $D$ is an open normal subgroup of $K$ to begin with. Then, by Lemma $1, \omega_{Y_{Q}}\left(E_{\pi}(D, T)\right)$ equals

$$
\frac{\left[D K^{Q}: D\right] C_{G} d_{Q}}{[K: D] C_{Q} d_{G}} \int_{0}^{T^{[k: \mathbb{Q}] / e_{\pi}}} t^{e_{Q}} \frac{d t}{t}=\frac{\left[D K^{Q}: D\right] C_{G} d_{Q}}{[K: D] C_{Q} d_{G} e_{Q}} T^{e_{Q}[k: \mathbb{Q}] / e_{\pi}} .
$$

Let $\chi_{T}$ be the characteristic function of $E_{\pi}(D, T)$. Define the function $F_{T}$ on $G(k) \backslash G(\mathbb{A})^{1}$ as

$$
F_{T}(g)=\frac{1}{\omega_{Y_{Q}}\left(E_{\pi}(D, T)\right)} \sum_{x \in X_{Q}(k)} \chi_{T}(x g)=\frac{\left|E_{\pi}(D, T) \cap X_{Q}(k) g\right|}{\omega_{Y_{Q}}\left(E_{\pi}(D, T)\right)} .
$$


(3.3) is equivalent to the assertion that

$$
\lim _{T \rightarrow \infty} F_{T}(g)=\frac{\tau(Q)}{\tau(G)}
$$

holds for every $g \in G(\mathbb{A})^{1}$. For a pair of functions $\psi_{1}, \psi_{2}$ on $G(k) \backslash G(\mathbb{A})^{1}$, we set

$$
\left\langle\psi_{1}, \psi_{2}\right\rangle=\int_{G(k) \backslash G(\mathbb{A})^{1}} \psi_{1}(g) \overline{\psi_{2}(g)} d \omega_{G}(g)
$$

if the integral has the meaning.

Proposition 1. If

$$
\lim _{T \rightarrow \infty}\left\langle\psi, F_{T}\right\rangle=\frac{\tau(Q)}{\tau(G)}\langle\psi, 1\rangle
$$

holds for any $\psi \in C_{0}\left(G(k) \backslash G(\mathbb{A})^{1}\right)$, then

$$
\lim _{T \rightarrow \infty} F_{T}(g)=\frac{\tau(Q)}{\tau(G)}
$$

for every $g \in G(\mathbb{A})^{1}$.

Proof. Let $\left\{U_{m}\right\}_{m=1,2,3, \ldots}$ be a descending family of neighborhoods of the identity $e$ in $G(\mathbb{A})^{1}$ such that $U_{m}$ is decomposable, i.e., $U_{m}=\left(U_{m}\right)_{\infty} \times$ $\left(U_{m}\right)_{f}, U_{m}^{-1}=U_{m},\left(U_{m}\right)_{f}=D_{f},\left(U_{m}\right)_{\infty}$ is compact and $\bigcap_{m=1}^{\infty}\left(U_{m}\right)_{\infty}=$ $\{e\}$. Since $\Phi_{\pi}$ is continuous and $K U_{m}$ is compact, there exists the maximum

$$
\beta_{m}=\max _{g \in K U_{m}} \Phi_{\pi}(g)=\max _{g_{\infty} \in K_{\infty}\left(U_{m}\right)_{\infty}} \Phi_{\pi}\left(g_{\infty}\right) .
$$

From the right $K$-invariance of $\Phi_{\pi}$ and $\Phi_{\pi}(e)=1$, it follows that $\beta_{m} \downarrow 1$ as $m \rightarrow \infty$. By $D_{\infty}=K_{\infty}$ and the definition of $E_{\pi}(D, T)$, it is evident that

$$
E_{\pi}(D, T) U_{m} \subset E_{\pi}\left(D, \beta_{m} T\right)
$$

for every $m$. Therefore,

$$
E_{\pi}\left(D, \beta_{m}^{-1} T\right) g^{-1} g_{0}^{-1} \subset E_{\pi}(D, T) g_{0}^{-1} \subset E_{\pi}\left(D, \beta_{m} T\right) g^{-1} g_{0}^{-1}
$$

holds for every $g \in U_{m}=U_{m}^{-1}$ and a fixed $g_{0} \in G(\mathbb{A})^{1}$. This implies the inequality

$$
\begin{aligned}
\omega_{Y_{Q}}\left(E_{\pi}\left(D, \beta_{m}^{-1} T\right)\right) F_{\beta_{m}^{-1} T}\left(g_{0} g\right) & \leq \omega_{Y_{Q}}\left(E_{\pi}(D, T)\right) F_{T}\left(g_{0}\right) \\
& \leq \omega_{Y_{Q}}\left(E_{\pi}\left(D, \beta_{m} T\right)\right) F_{\beta_{m} T}\left(g_{0} g\right)
\end{aligned}
$$


for $g \in U_{m}$. Let $U_{m}^{\prime}$ be the image of $g_{0} U_{m}$ to the quotient $G(k) \backslash G(\mathbb{A})^{1}$. We choose a real-valued and non-negative function $\psi_{m} \in C_{0}\left(G(k) \backslash G(\mathbb{A})^{1}\right)$ such that the support of $\psi_{m}$ is contained in $U_{m}^{\prime}$ and $\left\langle\psi_{m}, 1\right\rangle=1$. Then the above inequality yields

$$
\begin{aligned}
\frac{\omega_{Y_{Q}}\left(E_{\pi}\left(D, \beta_{m}^{-1} T\right)\right)}{\omega_{Y_{Q}}\left(E_{\pi}(D, T)\right)}\left\langle\psi_{m}, F_{\beta_{m}^{-1} T}\right\rangle & \leq F_{T}\left(g_{0}\right) \\
& \leq \frac{\omega_{Y_{Q}}\left(E_{\pi}\left(D, \beta_{m} T\right)\right)}{\omega_{Y_{Q}}\left(E_{\pi}(D, T)\right)}\left\langle\psi_{m}, F_{\beta_{m} T}\right\rangle .
\end{aligned}
$$

By $\omega_{Y_{Q}}\left(E_{\pi}\left(D, \beta_{m} T\right)\right) / \omega_{Y_{Q}}\left(E_{\pi}(D, T)\right)=\beta_{m}^{e_{Q}[k: \mathbb{Q}] / e_{\pi}}$ and the assumption on $F_{T}$, one has

$$
\beta_{m}^{-e_{Q}[k: \mathbb{Q}] / e_{\pi}} \frac{\tau(Q)}{\tau(G)} \leq \liminf _{T \rightarrow \infty} F_{T}\left(g_{0}\right) \leq \limsup _{T \rightarrow \infty} F_{T}\left(g_{0}\right) \leq \beta_{m}^{e_{Q}[k: \mathbb{Q}] / e_{\pi}} \frac{\tau(Q)}{\tau(G)} .
$$

Hence, letting $m \rightarrow \infty$, we get the assertion.

For every function $\psi$ on $G(k) \backslash G(\mathbb{A})^{1}$, we set

$$
\begin{aligned}
\Pi_{Q}^{1}(\psi)(g) & =\int_{U_{Q}(k) \backslash U_{Q}(\mathbb{A})} \psi(u g) d \omega_{U_{Q}}(u), \\
\Pi_{Q}(\psi)(g) & =\int_{Q(k) \backslash Q(\mathbb{A})^{1}} \psi(q g) d \omega_{Q}(q) \\
& =\int_{M_{Q}(k) \backslash M_{Q}(\mathbb{A})^{1}} \Pi_{Q}^{1}(\psi)(m g) d \omega_{M_{Q}}(m)
\end{aligned}
$$

when the integrals have the meaning. By the unfolding argument and Lemma 1, we have

$$
\begin{aligned}
\left\langle\psi, F_{T}\right\rangle & =\int_{G(k) \backslash G(\mathbb{A})^{1}} \psi(g) F_{T}(g) d \omega_{G}(g) \\
& =\frac{1}{\omega_{Y_{Q}}\left(E_{\pi}(D, T)\right)} \int_{Y_{Q}} \Pi_{Q}(\psi)(y) \chi_{T}(y) d \omega_{Y_{Q}}(y) \\
& =\frac{e_{Q}}{T^{e_{Q}[k: \mathbb{Q}] / e_{\pi}}} \int_{0}^{T^{[k: \mathbb{Q}] / e_{\pi}}} t^{e_{Q}} \Pi_{Q}(\psi)\left(\iota_{Q}\left(\bar{e},\left|\alpha_{Q}\right|_{\mathbb{A}}^{-1}(t)\right)\right) \frac{d t}{t}
\end{aligned}
$$

for every right $D$-invariant $\psi \in C_{0}\left(G(k) \backslash G(\mathbb{A})^{1}\right)$, where $\left|\alpha_{Q}\right|_{\mathbb{A}}^{-1}$ stands for the inverse map of $\left|\alpha_{Q}\right|_{\mathbb{A}}: A_{Q}^{G} \rightarrow \mathbb{R}_{+}^{\times}$. 


\section{§4. Preliminaries on Eisenstein series}

We recall the theory of Eisenstein series following [H], [MW]. Let $R$ be a standard $k$-parabolic subgroup of $G$. We set

$$
\operatorname{Re} \mathfrak{a}_{R}=X^{*}\left(Z_{G} \backslash Z_{R}\right) \otimes_{\mathbb{Z}} \mathbb{R}, \quad \mathfrak{a}_{R}=\operatorname{Re} \mathfrak{a}_{R} \otimes_{\mathbb{R}} \mathbb{C}=\operatorname{Re} \mathfrak{a}_{R}+\sqrt{-1} \operatorname{Re} \mathfrak{a}_{R} .
$$

Every $\Lambda \in \mathfrak{a}_{R}$ of the form $\chi_{1} \otimes s_{1}+\cdots+\chi_{r} \otimes s_{r}, \chi_{i} \in X^{*}\left(Z_{G} \backslash Z_{R}\right), s_{i} \in \mathbb{C}$ gives rise to a quasi-character of $A_{R}^{G}$ by

$$
z \longmapsto z^{\Lambda}=\left|\chi_{1}(z)\right|_{\mathbb{A}}^{s_{1}} \cdots\left|\chi_{r}(z)\right|_{\mathbb{A}}^{s_{r}}
$$

for $z \in A_{R}^{G}$. By this way, $\mathfrak{a}_{R}$ is identified with the group of quasi-characters of $A_{R}^{G}$. There is a unique $\rho_{R} \in \operatorname{Re} \mathfrak{a}_{R}$ such that $z^{2 \rho_{R}}=\delta_{R}(z)$. If $R^{\prime}$ is a standard $k$-parabolic subgroup of $G$ such that $R^{\prime} \subset R$, then $Z_{G} \backslash Z_{R}$ (resp. $A_{R}^{G}$ ) is a subgroup of $Z_{G} \backslash Z_{R^{\prime}}$ (resp. $A_{R^{\prime}}^{G}$ ) and hence there is a natural surjection from $\mathfrak{a}_{R^{\prime}}$ onto $\mathfrak{a}_{R}$. The kernel of this surjection is denoted by $\mathfrak{a}_{R^{\prime}}^{R}$. Since the quasi-characters of $M_{R}(\mathbb{A})^{1} \backslash M_{R}(\mathbb{A})$ is restricted to $M_{R^{\prime}}(\mathbb{A})^{1} \backslash M_{R^{\prime}}(\mathbb{A})$ ([MW, I.1.4.(2)]), there is a splitting $\mathfrak{a}_{R} \rightarrow \mathfrak{a}_{R^{\prime}}$, and hence a direct product decomposition: $\mathfrak{a}_{R^{\prime}}=\mathfrak{a}_{R} \oplus \mathfrak{a}_{R^{\prime}}^{R}$. The subspace $\mathfrak{a}_{R^{\prime}}^{R}$ is identified with the group of quasi-characters of $A_{R^{\prime}}^{R}=A_{R^{\prime}} / A_{R}$ by the similar way as above. If $\left(\delta_{R^{\prime}}^{R}\right)^{-1}$ denotes the modular character of $\left(M_{R} \cap R^{\prime}\right)(\mathbb{A})$, there is a unique $\rho_{R^{\prime}}^{R} \in \operatorname{Re} \mathfrak{a}_{R^{\prime}}^{R}$ such that $z^{2 \rho_{R^{\prime}}^{R}}=\delta_{R^{\prime}}^{R}(z)$ for $z \in A_{R^{\prime}}^{R}$. One has $\rho_{R^{\prime}}=\rho_{R}+\rho_{R^{\prime}}^{R}$. We always consider $\mathfrak{a}_{R}$ as a subspace of $\mathfrak{a}_{P}$ and fix an admissible inner product $(\cdot, \cdot)$ on $\operatorname{Re} \mathfrak{a}_{P}$. Then $\operatorname{Re} \mathfrak{a}_{R^{\prime}}=\operatorname{Re} \mathfrak{a}_{R} \oplus \operatorname{Re} \mathfrak{a}_{R^{\prime}}^{R}$ is an orthogonal decomposition. For each root $\beta \in \Phi_{k}, \beta^{\vee}$ denotes the coroot $2(\beta, \beta)^{-1} \beta$. Let $\Delta_{R}$ denote the set consisting of nonzero $\left.\operatorname{roots} \beta\right|_{Z_{R}}, \beta \in \Delta_{k}$. It is obvious that $\Delta_{R}$ is contained in $\operatorname{Re} \mathfrak{a}_{R}$ and spans $\mathfrak{a}_{R}$ as a $\mathbb{C}$-vector space. We set

$$
\mathfrak{c}_{R}=\left\{\Lambda \in \mathfrak{a}_{R}:\left(\operatorname{Re} \Lambda-\rho_{R},\left.\beta^{\vee}\right|_{Z_{R}}\right)>0 \text { for all }\left.\beta\right|_{Z_{R}} \in \Delta_{R}\right\}
$$

and

$$
\begin{aligned}
\mathfrak{c}_{R^{\prime}}^{R}=\left\{\Lambda \in \mathfrak{a}_{R^{\prime}}^{R}:\left(\operatorname{Re} \Lambda-\rho_{R^{\prime}}^{R},\left.\beta^{\vee}\right|_{Z_{R^{\prime}}}\right)>0 \text { for all }\left.\beta\right|_{Z_{R^{\prime}}}\right. & \in \Delta_{R^{\prime}} \\
& \text { with } \left.\left.\beta\right|_{Z_{R}}=0\right\} .
\end{aligned}
$$

A map $z_{R}: G(\mathbb{A}) \rightarrow A_{R}^{G}=A_{G} M_{R}(\mathbb{A})^{1} \backslash M_{R}(\mathbb{A})$ is defined by $z_{R}(g)=$ $A_{G} M_{R}(\mathbb{A})^{1} m$ if $g=u m h, u \in U_{R}(\mathbb{A}), m \in M_{R}(\mathbb{A})$ and $h \in K$.

For a smooth function $\eta \in C_{0}^{\infty}\left(A_{R}^{G}\right)$, its Mellin transform is defined to be

$$
\widehat{\eta}(\Lambda)=\int_{A_{R}^{G}} \eta(z) z^{-\left(\Lambda+\rho_{R}\right)} d \nu_{A_{R}^{G}}(z) .
$$


We choose the measure $d \Lambda$ on $\mathfrak{a}_{R}$ so that the following inversion formula holds for any $\eta \in C_{0}^{\infty}\left(A_{R}^{G}\right)$ :

$$
\eta(z)=\int_{\Lambda \in \Lambda_{0}+\sqrt{-1} \operatorname{Re} \mathfrak{a}_{R}} \widehat{\eta}(\Lambda) z^{\Lambda+\rho_{R}} d \Lambda,
$$

where $\Lambda_{0} \in \operatorname{Re} \mathfrak{a}_{R}$ is a base point.

Let $\mathcal{A}_{0, R}=\mathcal{A}_{0}\left(A_{R}^{G} U_{R}(\mathbb{A}) M_{R}(k) \backslash G(\mathbb{A})^{1}\right)$ be the space of cuspidal automorphic forms on $A_{R}^{G} U_{R}(\mathbb{A}) M_{R}(k) \backslash G(\mathbb{A})^{1}$. For an open subgroup $D \subset K$, $\mathcal{A}_{0, R}^{D}$ denotes the set of right $D$-invariant cusp forms in $\mathcal{A}_{0, R}$. For $\varphi \in \mathcal{A}_{0, R}$, $\eta \in C_{0}^{\infty}\left(A_{R}^{G}\right)$ and $\Lambda \in \mathfrak{c}_{R}$, the pseudo-Eisenstein series $\theta_{\varphi, \eta}$ and the Eisenstein series $E(\varphi, \Lambda)$ on $G(k) \backslash G(\mathbb{A})^{1}$ are defined as follows:

$$
\begin{aligned}
\theta_{\varphi, \eta}(g) & =\sum_{\gamma \in R(k) \backslash G(k)} \varphi(\gamma g) \eta\left(z_{R}(\gamma g)\right), \\
E(\varphi, \Lambda)(g) & =\sum_{\gamma \in R(k) \backslash G(k)} z_{R}(\gamma g)^{\Lambda+\rho_{R}} \varphi(\gamma g) .
\end{aligned}
$$

It is known that both series are absolutely convergent, $\theta_{\varphi, \eta}$ is a rapidly decreasing function on $G(k) \backslash G(\mathbb{A})^{1}$ and $E(\varphi, \Lambda)$ is meromorphically continued on the whole $\mathfrak{a}_{R}$. If $\Lambda_{0} \in \operatorname{Re} \mathfrak{a}_{R} \cap \mathfrak{c}_{R}$ is fixed, then $\theta_{\varphi, \eta}$ is expressed as

$$
\theta_{\varphi, \eta}(g)=\int_{\Lambda \in \Lambda_{0}+\sqrt{-1} \operatorname{Re} \mathfrak{a}_{R}} \widehat{\eta}(\Lambda) E(\varphi, \Lambda)(g) d \Lambda .
$$

We need intertwining operators to describe constant terms of pseudoEisenstein series. Let $W_{G}$ be the relative Weyl groups of $(G, S)$. We take a pair of a standard $k$-parabolic subgroup $R^{\prime}$ and an element $w \in W_{G}$ such that $w M_{R} w^{-1}=M_{R^{\prime}}$. Then, for $\Lambda \in \mathfrak{c}_{R}$ and $\varphi \in \mathcal{A}_{0, R}$, we consider

$$
\begin{aligned}
& (M(w, \Lambda) \varphi)(g)=z_{R^{\prime}}(g)^{-\left(w \Lambda+\rho_{R^{\prime}}\right)} \\
& \quad \times \int_{\left(U_{R^{\prime}}(\mathbb{A}) \cap w U_{R}(\mathbb{A}) w^{-1}\right) \backslash U_{R^{\prime}}(\mathbb{A})} \varphi\left(w^{-1} u g\right) z_{R}\left(w^{-1} u g\right)^{\Lambda+\rho_{R}} d \omega_{\mathbb{A}}^{U_{R^{\prime}}}(u) .
\end{aligned}
$$

The integral of the right-hand side converges absolutely and $M(w, \Lambda) \varphi$ is contained in $\mathcal{A}_{0, R^{\prime}}$. Moreover, the operator $M(w, \Lambda)$ is meromorphically continued to the whole $\mathfrak{a}_{R}$. The adjoint operator $M(w, \Lambda)^{*}$ of $M(w, \Lambda)$ with respect to the $L^{2}$-inner product on $\mathcal{A}_{0, R}$ equals $M\left(w^{-1},-w \bar{\Lambda}\right)$. 


\section{§5. Proof of Theorem 1}

Let $\pi, Q, D$ and $F_{T}$ be the same as in Section 3. On account of Proposition 1, we must prove

$$
\lim _{T \rightarrow \infty}\left\langle\psi, F_{T}\right\rangle=\frac{\tau(Q)}{\tau(G)}\langle\psi, 1\rangle
$$

for every $\psi \in C_{0}(G(k) \backslash G(\mathbb{A}))$. By [DRS, Lemma 2.4], it is enough to prove

$$
\lim _{T \rightarrow \infty}\left\langle\theta_{\varphi, \eta}, F_{T}\right\rangle=\frac{\tau(Q)}{\tau(G)}\left\langle\theta_{\varphi, \eta}, 1\right\rangle
$$

for all pseudo-Eisenstein series $\theta_{\varphi, \eta}$.

Proposition 2. Let $R$ be a standard k-parabolic subgroup of $G, \varphi \in$ $\mathcal{A}_{0, R}$ and $\eta \in C_{0}^{\infty}\left(A_{R}^{G}\right)$. If $R \neq P$, i.e., $R$ is not a minimal k-parabolic subgroup, then

$$
\left\langle\theta_{\varphi, \eta}, F_{T}\right\rangle=\left\langle\theta_{\varphi, \eta}, 1\right\rangle=0 .
$$

Proof. First, by (1.3) and $\omega_{G(\mathbb{A})^{1}}=\left(d_{G} \mu_{A_{G}}\right) \backslash \omega_{\mathbb{A}}^{G}$, one has

$$
\begin{aligned}
\left\langle\theta_{\varphi, \eta}, 1\right\rangle= & \int_{R(k) \backslash G(\mathbb{A})^{1}} \varphi(g) \eta\left(z_{R}(g)\right) d\left(\omega_{R(k)} \backslash \omega_{G(\mathbb{A})^{1}}\right)(g) \\
= & \frac{C_{G}}{C_{R} d_{G}} \int_{U_{R}(k) \backslash U_{R}(\mathbb{A}) \times A_{G} M_{R}(k) \backslash M_{R}(\mathbb{A}) \times K} \varphi(m h) \eta\left(z_{R}(m)\right) \\
& \times \delta_{R}(m)^{-1} d \omega_{U_{R}}(u) d\left(\mu_{A_{G}} \omega_{G(k)} \backslash \omega_{\mathbb{A}}^{M_{R}}\right)(m) d \nu_{K}(h) \\
= & \frac{C_{G} d_{R}}{C_{R} d_{G}} \int_{M_{R}(k) \backslash M_{R}(\mathbb{A})^{1} \times K} \varphi(m h)\left\{\int_{A_{R}^{G}} \eta(z) z^{-2 \rho_{R}} d \nu_{A_{R}^{G}}(z)\right\} \\
\quad \times d \omega_{M_{R}}(m) d \nu_{K}(h) & \\
= & \frac{C_{G} d_{R}}{C_{R} d_{G}} \widehat{\eta}\left(\rho_{R}\right)\langle\varphi, 1\rangle_{R},
\end{aligned}
$$

where we set

$$
\langle\varphi, 1\rangle_{R}=\int_{M_{R}(k) \backslash M_{R}(\mathbb{A})^{1} \times K} \varphi(m h) d \omega_{M_{R}}(m) d \nu_{K}(h) .
$$

From the cuspidality of $\varphi$, it follows $\langle\varphi, 1\rangle_{R}=0$, and hence $\left\langle\theta_{\varphi, \eta}, 1\right\rangle=0$. 
Next we compute $\Pi_{Q}\left(\theta_{\varphi, \eta}\right)$. Since $Q$ is maximal, there is an only one simple root $\alpha \in \Delta_{k}$ such that $\left.\alpha\right|_{Z_{Q}} \neq 0$. We define a subset $W\left(M_{R}, M_{Q}\right)$ of the Weyl group $W_{G}$ by

$$
\begin{array}{r}
W\left(M_{R}, M_{Q}\right)=\left\{w \in W_{G}: w^{-1}(\beta)>0 \text { for all } \beta \in \Delta_{k}-\{\alpha\}\right. \\
\text { and } \left.w R w^{-1} \subset Q\right\} .
\end{array}
$$

Then the constant term of the Eisenstein series $E(\varphi, \Lambda)$ along $U_{Q}$ is given by the formula

$$
\begin{aligned}
& \Pi_{Q}^{1}(E(\varphi, \Lambda))(g) \\
& \quad=\sum_{w \in W\left(M_{R}, M_{Q}\right)} \sum_{\gamma \in M_{Q}(k) \cap R^{w}(k) \backslash M_{Q}(k)}(M(w, \Lambda) \varphi)(\gamma g) z_{R^{w}}(\gamma g)^{w \Lambda+\rho_{R^{w}}},
\end{aligned}
$$

where $R^{w}$ denotes $w R w^{-1}$ ([MW, Proposition II.1.7]). If $W\left(M_{R}, M_{Q}\right)$ is empty, this constant term is zero. Thus $\Pi_{Q}^{1}\left(\theta_{\varphi, \eta}\right)(g)$ equals

$$
\begin{aligned}
& \sum_{w \in W\left(M_{R}, M_{Q}\right)} \int_{\Lambda \in \Lambda_{0}+\sqrt{-1} \operatorname{Re} \mathfrak{a}_{R}} \widehat{\eta}(\Lambda) \\
& \quad \times \sum_{\gamma \in M_{Q}(k) \cap R^{w}(k) \backslash M_{Q}(k)}(M(w, \Lambda) \varphi)(\gamma g) z_{R^{w}}(\gamma g)^{w \Lambda+\rho_{R^{w}}} d \Lambda \\
& =\sum_{w \in W\left(M_{R}, M_{Q}\right)} \int_{\Lambda \in w \Lambda_{0}+\sqrt{-1} \operatorname{Re}_{R^{w}}} \widehat{\eta}\left(w^{-1} \Lambda\right) \\
& \quad \times \sum_{\gamma \in M_{Q}(k) \cap R^{w}(k) \backslash M_{Q}(k)}\left(M\left(w, w^{-1} \Lambda\right) \varphi\right)(\gamma g) z_{R^{w}}(\gamma g)^{\Lambda+\rho_{R^{w}}} d \Lambda .
\end{aligned}
$$

We take $m \in A_{G} \backslash M_{Q}(\mathbb{A})$ and $m_{1} \in M_{Q}(\mathbb{A})^{1}$ so that $m=m_{1} z_{Q}(m)$. Then one has $z_{R^{w}}(\gamma m)=z_{Q}(m) z_{R^{w}}\left(\gamma m_{1}\right)$ and $z_{R^{w}}(\gamma m)^{\Lambda}=z_{Q}(m)^{\Lambda_{1}} z_{R^{w}}\left(\gamma m_{1}\right)^{\Lambda_{2}}$ for $\Lambda=\Lambda_{1}+\Lambda_{2}, \Lambda_{1} \in \mathfrak{a}_{Q}$ and $\Lambda_{2} \in \mathfrak{a}_{R^{w}}^{Q}$ because of $\gamma m_{1} \in M_{Q}(\mathbb{A})^{1}$. We choose a base point $\Lambda_{1,0} \in \operatorname{Re} \mathfrak{a}_{Q}$ and $\Lambda_{w, 0} \in \operatorname{Re} \mathfrak{a}_{R^{w}}^{Q}$ as follows: $\left(-\Lambda_{1,0},\left.\alpha^{\vee}\right|_{Z_{Q}}\right)$ is sufficiently large, and $\left(\Lambda_{w, 0}-\rho_{R^{w}}^{Q},\left.\beta^{\vee}\right|_{Z_{R^{w}}}\right)>0$ for all $\left.\beta\right|_{Z_{R^{w}}} \in \Delta_{R^{w}}$ with $\left.\beta\right|_{Z_{Q}}=0$. Then we can shift the integral domain of (5.2) from $w \Lambda_{0}+\sqrt{-1} \operatorname{Re} \mathfrak{a}_{R^{w}}$ to $\Lambda_{1,0}+\Lambda_{w, 0}+\sqrt{-1} \operatorname{Re} \mathfrak{a}_{R^{w}}$ ([MW, Lemma II.2.2]). 
Summing up, (5.2) at $g=m$ is equal to

$$
\begin{aligned}
\sum_{w \in W\left(M_{R}, M_{Q}\right)} \int_{\Lambda_{1} \in \Lambda_{1,0}+\sqrt{-1} \operatorname{Re} \mathfrak{a}_{Q}} z_{Q}(m)^{\Lambda_{1}+\rho_{Q}} \\
\times \sum_{\gamma \in M_{Q}(k) \cap R^{w}(k) \backslash M_{Q}(k)} \Psi_{w}\left(\Lambda_{1}, \gamma m_{1}\right) d \Lambda_{1},
\end{aligned}
$$

where

$$
\begin{aligned}
\Psi_{w}\left(\Lambda_{1}, m_{1}\right)= & \int_{\Lambda_{2} \in \Lambda_{w, 0}+\sqrt{-1} \operatorname{Re} \mathfrak{a}_{R^{w}}^{Q}} \widehat{\eta}\left(w^{-1}\left(\Lambda_{1}+\Lambda_{2}\right)\right) \\
& \times\left(M\left(w, w^{-1}\left(\Lambda_{1}+\Lambda_{2}\right)\right) \varphi\right)\left(m_{1}\right) z_{R^{w}}\left(m_{1}\right)^{\Lambda_{2}+\rho_{R}^{Q}} d \Lambda_{2} .
\end{aligned}
$$

Therefore, for $z \in A_{Q}^{G}$,

$$
\begin{aligned}
\Pi_{Q}\left(\theta_{\varphi, \eta}\right)(z) & \int_{M_{Q}(k) \backslash M_{Q}(\mathbb{A})^{1}} \Pi_{Q}^{1}\left(\theta_{\varphi, \eta}\right)\left(m_{1} z\right) d \omega_{M_{Q}}\left(m_{1}\right) \\
= & \left.\sum_{w \in W\left(M_{R}, M_{Q}\right)} \int_{\Lambda_{1} \in \Lambda_{1,0}+\sqrt{-1} \operatorname{Re}_{Q} z^{\Lambda_{1}+\rho_{Q}}} \sum_{\gamma \in M_{Q}(k) \cap R^{w}(k) \backslash M_{Q}(k)} \Psi_{w}\left(\Lambda_{1}, \gamma m_{1}\right) d \omega_{M_{Q}}\left(m_{1}\right)\right\} d \Lambda_{1} .
\end{aligned}
$$

By the calculation similar to (5.1), the inner integral equals

$$
\begin{aligned}
& \frac{C_{Q} d_{R^{w}}}{C_{R^{w}} d_{Q}} \int_{A_{R^{w}}^{Q}}\left\{\int_{M_{R^{w}}(k) \backslash M_{R^{w}}(\mathbb{A})^{1} \times K^{M_{Q}}} \Psi_{w}\left(\Lambda_{1}, z_{2} m_{2} h\right)\right. \\
& \left.\times d \omega_{M_{R^{w}}}\left(m_{2}\right) d \nu_{K^{M_{Q}}}(h)\right\}\left(\delta_{R^{w}}^{Q}\right)^{-1}\left(z_{2}\right) d\left(\mu_{A_{Q}} \backslash \mu_{A_{R^{w}}}\right)\left(z_{2}\right) \\
& =\frac{C_{Q} d_{R^{w}}}{C_{R^{w}} d_{Q}} \int_{A_{R^{w}}^{Q}} \int_{\Lambda_{2} \in \Lambda_{w, 0}+\sqrt{-1} \operatorname{Re} \mathfrak{a}_{R^{w}}^{Q}} \widehat{\eta}\left(w^{-1}\left(\Lambda_{1}+\Lambda_{2}\right)\right) \\
& \times\left\{\int_{M_{R^{w}}(k) \backslash M_{R^{w}}(\mathbb{A})^{1} \times K^{M_{Q}}}\left(M\left(w, w^{-1}\left(\Lambda_{1}+\Lambda_{2}\right)\right) \varphi\right)\left(m_{2} h\right)\right. \\
& \left.\times d \omega_{M_{R^{w}}}\left(m_{2}\right) d \nu_{K^{M_{Q}}}(h)\right\} z_{2}^{\Lambda_{2}-\rho_{R^{w}}^{Q}} d \Lambda_{2} d\left(\mu_{A_{Q}} \backslash \mu_{A_{R^{w}}}\right)\left(z_{2}\right)
\end{aligned}
$$


The cuspidality of $M\left(w, w^{-1} \Lambda\right) \varphi$ implies

$$
\int_{M_{R^{w}}(k) \backslash M_{R} w(\mathbb{A})^{1} \times K^{M_{Q}}}\left(M\left(w, w^{-1} \Lambda\right) \varphi\right)\left(m_{2} h\right) d \omega_{M_{R} w}\left(m_{2}\right) d \nu_{K^{M_{Q}}}(h)=0 .
$$

Hence $\left.\Pi_{Q}\left(\theta_{\varphi, \eta}\right)\right|_{M_{Q}(\mathbb{A})} \equiv 0$. This implies $\left\langle\theta_{\varphi, \eta}, F_{T}\right\rangle=0$ by $(3.4)$.

Next, we consider the case $R=P$. Since $P$ is a minimal $k$-parabolic subgroup, the constant function $\varphi_{0} \equiv 1$ is contained in $\mathcal{A}_{0, P}$. We define the inner product on $\mathcal{A}_{0, P}^{K}=\mathcal{A}_{0}\left(M(k) \backslash M(\mathbb{A})^{1}\right)^{K^{M}}$ by

$$
\left\langle\psi_{1}, \psi_{2}\right\rangle_{M}=\int_{M(k) \backslash M(\mathbb{A})^{1}} \psi_{1}(m) \overline{\psi_{2}(m)} d \omega_{M}(m) \quad\left(\psi_{1}, \psi_{2} \in \mathcal{A}_{0, P}^{K}\right) .
$$

Let $W_{M_{Q}}$ be the relative Weyl group of $\left(M_{Q}, S\right)$. As a subgroup of $W_{G}$, $W_{M_{Q}}$ is identified with the point wise stabilizer of $\mathfrak{a}_{Q}$ in $W_{G}$. For $w \in W_{G}$ and a generic $\Lambda \in \mathfrak{a}_{P}$, the operator $M(w, \Lambda)$ maps $\mathcal{A}_{0, P}^{D K^{Q}}$ into itself. If $w \in$ $W_{M_{Q}}$, then the equality $M\left(w, \Lambda_{1}+\Lambda_{2}\right)=M\left(w, \Lambda_{2}\right)$ holds for $\Lambda_{1} \in \mathfrak{a}_{Q}, \Lambda_{2} \in$ $\mathfrak{a}_{P}^{Q}$, and $M\left(w, \Lambda_{2}\right)$ is regarded as an operator on $\mathcal{A}_{0}\left(A_{P}^{Q} U(\mathbb{A}) M(k) \backslash Q(\mathbb{A})^{1}\right)$. We denote by $w_{0}$ (resp. $\left.w_{1}\right)$ the longest element of $W_{G}\left(\operatorname{resp} . W_{M_{Q}}\right)$. It is known from the theory of local intertwining operators and the Langlands classification theorem that the residue

$$
M\left(w_{0}\right)=\lim _{\substack{\Lambda \in \mathfrak{c}_{P} \\ \Lambda \rightarrow \rho_{P}}}\left(\prod_{\beta \in \Delta_{k}}\left(\Lambda-\rho_{P}, \beta^{\vee}\right)\right) M\left(w_{0}, \Lambda\right)
$$

exists and yields a projection from $\mathcal{A}_{0, P}$ onto the trivial representation $\mathbb{C} \varphi_{0}$ of $G(\mathbb{A})^{1}$ ([FMT, Section $\left.\left.10(\mathrm{~b})\right]\right)$. By the argument of [L] or [Lai], one has

$$
M\left(w_{0}\right) \varphi_{0}=\frac{C_{G} d_{P} \tau(P)}{d_{G} \tau(G)} \varphi_{0} .
$$

In a similar fashion, the residue

$$
M\left(w_{1}\right)=\lim _{\substack{\Lambda_{2} \in \mathfrak{c}_{P}^{Q} \\ \Lambda_{2} \rightarrow \rho_{P}^{Q}}}\left(\prod_{\beta \in \Delta_{k}-\{\alpha\}}\left(\Lambda_{2}-\rho_{P}^{Q}, \beta^{\vee}\right)\right) M\left(w_{1}, \Lambda_{2}\right)
$$

yields a projection from $\mathcal{A}_{0}\left(A_{P}^{Q} U(\mathbb{A}) M(k) \backslash Q(\mathbb{A})^{1}\right)$ onto $\mathbb{C} \varphi_{0}$ and one has

$$
M\left(w_{1}\right) \varphi_{0}=\frac{C_{Q} d_{P} \tau(P)}{d_{Q} \tau(Q)} \varphi_{0}
$$


Lemma 2. For any $\varphi \in \mathcal{A}_{0, P}$,

$$
M\left(w_{0}\right) \varphi=\frac{C_{G} d_{P}}{d_{G} \tau(G)}\langle\varphi, 1\rangle_{P} \varphi_{0}
$$

Proof. If $M\left(w_{0}\right) \varphi=c \varphi_{0}$, then

$$
c=\frac{1}{\tau(P)}\left\langle M\left(w_{0}\right) \varphi, \varphi_{0}\right\rangle_{P}=\frac{1}{\tau(P)}\left\langle\varphi, M\left(w_{0}\right)^{*} \varphi_{0}\right\rangle_{P}=\frac{C_{G} d_{P}}{d_{G} \tau(G)}\left\langle\varphi, \varphi_{0}\right\rangle_{P} .
$$

Here note that the constant $C_{G} d_{P} /\left(d_{G} \tau(G)\right)$ is a positive real value.

Lemma 3. Let $\tau \in W\left(M, M_{Q}\right), \sigma=\tau^{-1} w_{1} \in W_{G}$ and $\varphi \in \mathcal{A}_{0, P}^{D K^{Q}}$. If we fix a $\Lambda_{1} \in \mathfrak{a}_{Q}$ with $\left(-\operatorname{Re} \Lambda_{1},\left.\alpha^{\vee}\right|_{Z_{Q}}\right) \gg 0$, then the function

$$
\Lambda_{2} \longmapsto\left\langle\left.\left(M\left(\tau, \tau^{-1}\left(\Lambda_{1}+\Lambda_{2}\right)\right) \varphi\right)\right|_{M(\mathbb{A})^{1}}, \varphi_{0}\right\rangle_{M}
$$

is holomorphic at $\Lambda_{2}=\rho_{P}^{Q}$. Moreover, one has

$$
\begin{aligned}
& \left\langle\left.\left(M\left(\tau, \tau^{-1}\left(\Lambda_{1}+\rho_{P}^{Q}\right)\right) \varphi\right)\right|_{M(\mathbb{A})^{1}}, \varphi_{0}\right\rangle_{M} \\
& \quad=\frac{d_{Q} \tau(Q)}{C_{Q} d_{P} \tau(P)}\left\langle\left.\left(M_{1}\left(\sigma^{-1}, \sigma\left(\Lambda_{1}-\rho_{P}^{Q}\right)\right) \varphi\right)\right|_{M(\mathbb{A})^{1}}, \varphi_{0}\right\rangle_{M}
\end{aligned}
$$

where $M_{1}\left(\sigma^{-1}, \sigma\left(\Lambda_{1}-\rho_{P}^{Q}\right)\right)$ is defined by

$$
\lim _{\substack{\Lambda_{2} \in \mathfrak{c}_{P}^{Q} \\ \Lambda_{2} \rightarrow \rho_{P}^{Q}}}\left(\prod_{\beta \in \Delta_{k}-\{\alpha\}}\left(\Lambda_{2}-\rho_{P}^{Q}, \beta^{\vee}\right)\right) M\left(\sigma^{-1}, \sigma\left(\Lambda_{1}-\Lambda_{2}\right)\right)
$$

Proof. By [MW, Lemma II.2.2], the function $M\left(\tau, \tau^{-1}\left(\Lambda_{1}+\Lambda_{2}\right)\right) \varphi$ in $\Lambda_{2}$ is holomorphic on the tube domain of the form $\left\{\Lambda_{2} \in \mathfrak{a}_{P}^{Q}:\left(\operatorname{Re} \Lambda_{2}, \operatorname{Re} \Lambda_{2}\right)\right.$ $\left.<c_{0}^{2}\right\}$, where $c_{0}$ is a positive real constant with $c_{0}^{2}>\left(\rho_{P}, \rho_{P}\right)$. By the functional equations of $M(w, \Lambda)$,

$$
\begin{aligned}
\left\langle\left.\left(M\left(\tau, \tau^{-1} \Lambda\right) \varphi\right)\right|_{M(\mathbb{A})^{1}}, \varphi_{0}\right\rangle_{M} \\
\quad=\left\langle\left.\left(M\left(w_{1}, w_{1}^{-1} \Lambda\right) M\left(\sigma^{-1}, \sigma w_{1}^{-1} \Lambda\right) \varphi\right)\right|_{M(\mathbb{A})^{1}, \varphi_{0}}\right\rangle_{M} \\
\quad=\left\langle\left.\left(M\left(\sigma^{-1}, \sigma w_{1}^{-1} \Lambda\right) \varphi\right)\right|_{M(\mathbb{A})^{1}}, M\left(w_{1}, w_{1}^{-1} \Lambda\right)^{*} \varphi_{0}\right\rangle_{M} \\
\quad=\left\langle\left.\left(M\left(\sigma^{-1}, \sigma w_{1}^{-1} \Lambda\right) \varphi\right)\right|_{M(\mathbb{A})^{1}}, M\left(w_{1}^{-1},-\bar{\Lambda}\right) \varphi_{0}\right\rangle_{M}
\end{aligned}
$$


Here we identify $\mathcal{A}_{0, P}^{K}$ with $\mathcal{A}_{0}\left(A_{P}^{Q} U(\mathbb{A}) M(k) \backslash Q(\mathbb{A})^{1}\right)^{K^{M_{Q}}}$ and regard $M\left(w_{1}, w_{1}^{-1} \Lambda\right)$ as an operator on it. Therefore,

$$
\left\langle\left.\left(M\left(\tau, \tau^{-1}\left(\Lambda_{1}+\rho_{P}^{Q}\right)\right) \varphi\right)\right|_{M(\mathbb{A})^{1}}, \varphi_{0}\right\rangle_{M}
$$

equals

$$
\begin{aligned}
& \left\langle\left.\left(M_{1}\left(\sigma^{-1}, \sigma\left(\Lambda_{1}-\rho_{P}^{Q}\right)\right) \varphi\right)\right|_{M(\mathbb{A})^{1}},\right. \\
& \left.\lim _{\substack{\Lambda_{2} \in \mathfrak{c}_{P}^{Q} \\
\Lambda_{2} \rightarrow \rho_{P}^{Q}}}{\overline{\left(\prod_{\beta \in \Delta_{k}-\{\alpha\}}\left(\Lambda_{2}-\rho_{P}^{Q}, \beta^{\vee}\right)\right)}}^{-1} M\left(w_{1}^{-1},-\bar{\Lambda}_{2}\right) \varphi_{0}\right\rangle_{M} .
\end{aligned}
$$

If we regard $\overline{M\left(w_{1}^{-1},-\bar{\Lambda}_{2}\right)}$ acting on $\mathbb{C} \varphi_{0}$ as a scalar valued function, then

$$
\begin{aligned}
& \lim _{\substack{\Lambda_{2} \in \mathfrak{c}_{P}^{Q} \\
\Lambda_{2} \rightarrow \rho_{P}^{Q}}}\left(\prod_{\beta \in \Delta_{k}-\{\alpha\}}\left(\Lambda_{2}-\rho_{P}^{Q}, \beta^{\vee}\right)\right)^{-1} \overline{M\left(w_{1}^{-1},-\bar{\Lambda}_{2}\right)} \\
&=\lim _{\substack{\Lambda_{2} \in \mathfrak{c}_{P}^{Q} \\
\Lambda_{2} \rightarrow \rho_{P}^{Q}}}\left(\prod_{\beta \in \Delta_{k}-\{\alpha\}}\left(\Lambda_{2}-\rho_{P}^{Q}, \beta^{\vee}\right)\right)^{-1} \overline{M\left(w_{1},-w_{1}^{-1} \bar{\Lambda}_{2}\right)}-1 \\
&={\overline{M\left(w_{1}\right)}}^{-1} .
\end{aligned}
$$

This implies the assertion.

LEMMA 4. Being the notation as above, one has

$$
\lim _{\substack{\Lambda_{1} \in-\mathfrak{c}_{Q} \\ \Lambda_{1} \rightarrow-\rho_{Q}}}\left(\Lambda_{1}+\rho_{Q}, \alpha^{\vee}\right) M_{1}\left(\sigma^{-1}, \sigma\left(\Lambda_{1}-\rho_{P}^{Q}\right)\right) \varphi= \begin{cases}M\left(w_{0}\right) \varphi & \left(\sigma=w_{0}\right) \\ 0 & \left(\sigma \neq w_{0}\right)\end{cases}
$$

If $0<\varepsilon$ is sufficiently small, then the function

$$
\Lambda_{1} \longmapsto\left\langle\left.\left(M_{1}\left(\sigma^{-1}, \sigma\left(\Lambda_{1}-\rho_{P}^{Q}\right)\right) \varphi\right)\right|_{M(\mathbb{A})^{1}}, \varphi_{0}\right\rangle_{M}
$$

is holomorphic on $\left\{\Lambda_{1} \in \mathfrak{a}_{Q}: 1-\epsilon<\left(\operatorname{Re} \Lambda_{1}, \rho_{Q}\right) /\left(\rho_{Q}, \rho_{Q}\right)<1\right\}$ with polynomial growth as $\left|\Im \Lambda_{1}\right| \rightarrow \infty$. 
Proof. For any $\psi \in \mathcal{A}_{0, P}^{D K^{Q}}$

$$
\begin{aligned}
& \left\langle\lim _{\substack{\Lambda_{1} \in-\mathfrak{c}_{Q} \\
\Lambda_{1} \rightarrow-\rho_{Q}}}\left(\Lambda_{1}+\rho_{Q}, \alpha^{\vee}\right) M_{1}\left(\sigma^{-1}, \sigma\left(\Lambda_{1}-\rho_{P}^{Q}\right)\right) \varphi, \psi\right\rangle_{P} \\
& \quad=\left\langle\varphi, \lim _{\substack{\Lambda_{1} \in-\mathfrak{c}_{Q} \\
\Lambda_{1} \rightarrow-\rho_{Q}}} \overline{\left(\Lambda_{1}+\rho_{Q}, \alpha^{\vee}\right)} M_{1}\left(\sigma^{-1}, \sigma\left(\Lambda_{1}-\rho_{P}^{Q}\right)\right)^{*} \psi\right\rangle_{P} \\
& \left.\quad=\left\langle\varphi, \lim _{\substack{\Lambda_{1} \in-\mathfrak{c}_{Q} \\
\Lambda_{1 \rightarrow-} \rightarrow \rho_{Q}}} \overline{\left(\Lambda_{1}+\rho_{Q}, \alpha^{\vee}\right)} M_{1}\left(\sigma,-\bar{\Lambda}_{1}+\rho_{P}^{Q}\right)\right) \psi\right\rangle_{P} \\
& =\left\langle\varphi, \lim _{\substack{\Lambda \in \mathfrak{c}_{P} \\
\Lambda \rightarrow \rho_{P}}} \overline{\left(\prod_{\beta \in \Delta_{k}}\left(\Lambda-\rho_{P}, \beta^{\vee}\right)\right)} M(\sigma, \bar{\Lambda}) \psi\right\rangle_{P} .
\end{aligned}
$$

It is known that

$$
\lim _{\substack{\Lambda \in \mathfrak{c}_{P} \\ \Lambda \rightarrow \rho_{P}}}\left(\prod_{\beta \in \Delta_{k}}\left(\Lambda-\rho_{P}, \beta^{\vee}\right)\right) M(\sigma, \Lambda)= \begin{cases}M\left(w_{0}\right) & \left(\sigma=w_{0}\right) \\ 0 & \left(\sigma \neq w_{0}\right)\end{cases}
$$

(cf. [FMT, Lemma 7]). By this and Lemma 2, the equalities

$$
\begin{aligned}
\left\langle M\left(w_{0}\right) \varphi, \psi\right\rangle_{P} & =\left\langle\varphi, M\left(w_{0}\right) \psi\right\rangle_{P} \\
& =\left\langle\lim _{\substack{\Lambda_{1} \in-\mathfrak{c}_{Q} \\
\Lambda_{1} \rightarrow-\rho_{Q}}}\left(\Lambda_{1}+\rho_{Q}, \alpha^{\vee}\right) M_{1}\left(\sigma^{-1}, \sigma\left(\Lambda_{1}-\rho_{P}^{Q}\right)\right) \varphi, \psi\right\rangle_{P}
\end{aligned}
$$

hold for all $\psi \in \mathcal{A}_{0, P}^{D K^{Q}}$. The remains of the assertion follows from $[\mathrm{H}$, Lemma 118].

Proposition 3. Let $\varphi \in \mathcal{A}_{0, P}$ and $\eta \in C_{0}^{\infty}\left(A_{P}^{G}\right)$. Then one has

$$
\lim _{T \rightarrow \infty}\left\langle\theta_{\varphi, \eta}, F_{T}\right\rangle=\frac{\tau(Q)}{\tau(P)}\left\langle\theta_{\varphi, \eta}, 1\right\rangle .
$$

Proof. It is sufficient to prove the assertion for right $D K^{Q_{\text {-invariant }}}$ $\varphi \in \mathcal{A}_{0, P}$. The calculations of $\left\langle\theta_{\varphi, \eta}, 1\right\rangle$ and $\Pi_{Q}\left(\theta_{\varphi, \eta}\right)$ are the same as in the proof of Proposition 2. We have

$$
\left\langle\theta_{\varphi, \eta}, 1\right\rangle=\frac{C_{G} d_{P}}{C_{P} d_{G}} \widehat{\eta}\left(\rho_{P}\right)\langle\varphi, 1\rangle_{P}
$$


We need a further calculation of $\Pi_{Q}\left(\theta_{\varphi, \eta}\right)$. Since $\varphi$ is right $D K^{Q}$-invariant, $\Pi_{Q}\left(\theta_{\varphi, \eta}\right)(z)$ equals

$$
\frac{C_{Q} d_{P}}{C_{P} d_{Q}} \sum_{\tau \in W\left(M, M_{Q}\right)} \int_{\Lambda_{1} \in \Lambda_{1,0}+\sqrt{-1} \operatorname{Re} \mathfrak{a}_{Q}} z^{\Lambda_{1}+\rho_{Q}} \widehat{f}_{\tau}\left(\Lambda_{1}\right) d \Lambda_{1},
$$

where

$$
\begin{aligned}
\widehat{f}_{\tau}\left(\Lambda_{1}\right)=\int_{A_{P}^{Q}} & \int_{\Lambda_{2} \in \Lambda_{\tau, 0}+\sqrt{-1} \operatorname{Re} \mathfrak{a}_{P}^{Q}} \widehat{\eta}\left(\tau^{-1}\left(\Lambda_{1}+\Lambda_{2}\right)\right) \\
& \times\left\langle\left.\left(M\left(\tau, \tau^{-1}\left(\Lambda_{1}+\Lambda_{2}\right)\right) \varphi\right)\right|_{\left.M(\mathbb{A})^{1}, \varphi_{0}\right\rangle_{M} z_{2}^{\Lambda_{2}-\rho_{P}^{Q}}}\right. \\
& \times d \Lambda_{2} d\left(\mu_{A_{Q}} \backslash \mu_{A_{P}}\right)\left(z_{2}\right) .
\end{aligned}
$$

If $\Lambda_{1} \in \Lambda_{1,0}+\sqrt{-1} \operatorname{Re} \mathfrak{a}_{Q}$ is fixed, the function

$$
\Lambda_{2} \longmapsto \widehat{\eta}\left(\tau^{-1}\left(\Lambda_{1}+\Lambda_{2}\right)\right)\left\langle\left.\left(M\left(\tau, \tau^{-1}\left(\Lambda_{1}+\Lambda_{2}\right)\right) \varphi\right)\right|_{M(\mathbb{A})^{1}}, \varphi_{0}\right\rangle_{M}
$$

is holomorphic on the tube domain $\left\{\Lambda_{2} \in \mathfrak{a}_{P}^{Q}:\left(\operatorname{Re} \Lambda_{2}, \operatorname{Re} \Lambda_{2}\right)<c_{0}^{2}\right\}$ as mentioned in the proof of Lemma 3 . We can take $\Lambda_{\tau, 0}$ in this domain. Then, from the inversion formula, it follows

$$
\widehat{f}_{\tau}\left(\Lambda_{1}\right)=\widehat{\eta}\left(\tau^{-1}\left(\Lambda_{1}+\rho_{P}^{Q}\right)\right)\left\langle\left(M\left(\tau, \tau^{-1}\left(\Lambda_{1}+\rho_{P}^{Q}\right)\right) \varphi\right)_{M(\mathbb{A})^{1}}, \varphi_{0}\right\rangle_{M} .
$$

We shift the integral domain in (5.3) from $\Lambda_{1,0}+\sqrt{-1} \operatorname{Re} \mathfrak{a}_{Q}$ to $(\epsilon-1) \rho_{Q}+$ $\sqrt{-1} \operatorname{Re} \mathfrak{a}_{Q}$, where $\epsilon$ is a sufficiently small positive number so that all $\widehat{f}_{\tau}$ are holomorphic on the domain $B_{\epsilon}=\left\{\Lambda_{1} \in \mathfrak{a}_{Q}: 1-2 \epsilon<\left(-\operatorname{Re} \Lambda_{1}, \rho_{Q}\right) /\left(\rho_{Q}, \rho_{Q}\right)\right.$ $<1\}$. Taking account the residue at $-\rho_{Q}$, we obtain

$$
\begin{aligned}
& \int_{\Lambda_{1} \in \Lambda_{1,0}+\sqrt{-1} \operatorname{Re} \mathfrak{a}_{Q}} z^{\Lambda_{1}+\rho_{Q}} \widehat{f}_{\tau}\left(\Lambda_{1}\right) d \Lambda_{1} \\
& \quad=\int_{\Lambda_{1} \in(\epsilon-1) \rho_{Q}+\sqrt{-1} \operatorname{Re} \mathfrak{a}_{Q}} z^{\Lambda_{1}+\rho_{Q}} \widehat{f}_{\tau}\left(\Lambda_{1}\right) d \Lambda_{1}+\operatorname{Res}_{\Lambda_{1}=-\rho_{Q}} \widehat{f}_{\tau}\left(\Lambda_{1}\right) .
\end{aligned}
$$

We write $f_{\tau}(z)$ for the first term. By Lemmas 2,3 and $4, \Pi_{Q}\left(\theta_{\varphi, \eta}\right)(z)$ equals

$$
\begin{aligned}
& \frac{C_{Q} d_{P}}{C_{P} d_{Q}} \sum_{\tau \in W\left(M, M_{Q}\right)} f_{\tau}(z)+\frac{C_{Q} d_{P}}{C_{P} d_{Q}} \cdot \frac{d_{Q} \tau(Q)}{C_{Q} d_{P} \tau(P)} \widehat{\eta}\left(\rho_{P}\right)\left\langle\left. M\left(w_{0}\right) \varphi\right|_{M(\mathbb{A})^{1}}, \phi_{0}\right\rangle_{M} \\
& \quad=\frac{C_{Q} d_{P}}{C_{P} d_{Q}} \sum_{\tau \in W\left(M, M_{Q}\right)} f_{\tau}(z)+\frac{C_{G} d_{P} \tau(Q)}{C_{P} d_{G} \tau(G)} \widehat{\eta}\left(\rho_{P}\right)\langle\varphi, 1\rangle_{P} .
\end{aligned}
$$


Here note that $\left\langle\varphi_{0}, \varphi_{0}\right\rangle_{M}=\tau(M)=\tau(P)$. Since $\widehat{\eta}$ is a function of Paley Wiener type and $\widehat{f}_{\tau}\left(\Lambda_{1}\right) / \widehat{\eta}\left(\tau^{-1}\left(\Lambda_{1}+\rho_{P}^{Q}\right)\right)$ is of polynomial growth on $B_{\epsilon}$ as $\left|\Im \Lambda_{1}\right| \rightarrow \infty$ by Lemma 4 , we have an estimate of the formula

$$
\left|f_{\tau}(z)\right| \leq z^{\epsilon \rho_{Q}} \int_{\sqrt{-1} \operatorname{Re} \mathfrak{a}_{Q}}\left|z^{\Lambda}\right|\left|\widehat{f}_{\tau}\left((\epsilon-1) \rho_{Q}+\Lambda\right)\right| d \Lambda \leq c_{1} z^{\epsilon \rho_{Q}},
$$

where $c_{1}$ is a constant depending on $\widehat{f}_{\tau}$. This implies

$$
\begin{aligned}
& \limsup _{T \rightarrow \infty} \frac{e_{Q}}{T^{e_{Q}[k: \mathbb{Q}] / e_{\pi}}} \int_{0}^{T^{[k: \mathbb{Q}] / e_{\pi}}} t^{e_{Q}}\left|f_{\tau}\left(\iota_{Q}\left(\bar{e},\left|\alpha_{Q}\right|_{\mathbb{A}}^{-1}(t)\right)\right)\right| \frac{d t}{t} \\
& \quad \leq \limsup _{T \rightarrow \infty} \frac{e_{Q}}{T^{e_{Q}[k: \mathbb{Q}] / e_{\pi}}} \int_{0}^{T^{[k: \mathbb{Q}] / e_{\pi}}} c_{1} t^{(1-\epsilon / 2) e_{Q}} \frac{d t}{t}=0 .
\end{aligned}
$$

As a consequence, we have

$$
\lim _{T \rightarrow \infty}\left\langle\theta_{\varphi, \eta}, F_{T}\right\rangle=\frac{C_{G} d_{P} \tau(Q)}{C_{P} d_{G} \tau(G)} \widehat{\eta}\left(\rho_{P}\right)\langle\varphi, 1\rangle_{P}=\frac{\tau(Q)}{\tau(G)}\left\langle\theta_{\varphi, \eta}, 1\right\rangle .
$$

This completes the proof of Proposition 3, and therefore we are led to Theorem 1.

\section{$\S 6 . \quad$ Error terms}

We give some estimates of error terms of (3.3).

LEMMA 5. Let $a>0$ be a constant. If

$$
\lim _{T \rightarrow \infty}\left\langle\psi, \frac{F_{T}-\tau(Q) / \tau(G)}{T^{a}}\right\rangle=0
$$

holds for any $\psi \in C_{0}\left(G(k) \backslash G(\mathbb{A})^{1}\right)$, then one has

$$
\lim _{T \rightarrow \infty} \frac{F_{T}(g)-\tau(Q) / \tau(G)}{T^{a}}=0
$$

for every $g \in G(\mathbb{A})^{1}$.

Proof. Using the same notations as in the proof of Proposition 1, we have

$$
\begin{aligned}
& \beta_{m}^{-a-e_{Q}[k: \mathbb{Q}] / e_{\pi}} \frac{\left\langle\psi_{m}, F_{\beta_{m}^{-1} T}-\tau(Q) / \tau(G)\right\rangle}{\left(\beta_{m}^{-1} T\right)^{a}}+\frac{\left(\beta_{m}^{-e_{Q}[k: \mathbb{Q}] / e_{\pi}}-1\right) \tau(Q) / \tau(G)}{T^{a}} \\
& \leq \frac{F_{T}\left(g_{0}\right)-\tau(Q) / \tau(G)}{T^{a}} \\
& \leq \beta_{m}^{a+e_{Q}[k: \mathbb{Q}] / e_{\pi}} \frac{\left\langle\psi_{m}, F_{\beta_{m} T}-\tau(Q) / \tau(G)\right\rangle}{\left(\beta_{m} T\right)^{a}}+\frac{\left(\beta_{m}^{e_{Q}[k: \mathbb{Q}] / e_{\pi}}-1\right) \tau(Q) / \tau(G)}{T^{a}}
\end{aligned}
$$


The assertion follows immediately from this.

By [DRS, Lemma 2.4] and Proposition 2, if

$$
\lim _{T \rightarrow \infty}\left\langle\theta_{\varphi, \eta}, \frac{F_{T}-\tau(Q) / \tau(G)}{T^{a}}\right\rangle=0
$$

holds for all $\theta_{\varphi, \eta}, \varphi \in \mathcal{A}_{0, P}^{D K^{Q}}, \eta \in C_{0}^{\infty}\left(A_{P}^{G}\right)$, then we get (6.1). Let $\epsilon_{0}$ be the superior of $\epsilon \in(0,1 / 2)$ such that all $M\left(\tau, \tau^{-1}\left(\Lambda_{1}+\delta_{P}^{Q}\right)\right), \tau \in W\left(M, M_{Q}\right)$ are holomorphic on $B_{\epsilon}$, where $B_{\epsilon}$ is the same as in the proof of Proposition 3. Then, for any $0<a<\epsilon_{0}$, we can shift the integral domain of (5.3) from $\Lambda_{1,0}+\sqrt{-1} \operatorname{Re} \mathfrak{a}_{Q}$ to $(2 a-1) \rho_{Q}+\sqrt{-1} \operatorname{Re} \mathfrak{a}_{Q}$ and the estimate similar to (5.4) leads to

$$
\lim _{T \rightarrow \infty} \frac{\left\langle F_{T}, f_{\tau}\right\rangle}{T^{(1-a) e_{Q}[k: \mathbb{Q}] / e_{\pi}}}=0 .
$$

Thus we proved the following.

Proposition 4. For any $0<a<\epsilon_{0}$, one has

$$
\left|E_{\pi}(D, T) \cap X_{Q}(k) g\right|=\frac{\tau(Q)}{\tau(G)} \omega_{Y_{Q}}\left(E_{\pi}(D, T)\right)+o\left(T^{(1-a) e_{Q}[k: \mathbb{Q}] / e_{\pi}}\right) .
$$

We note that, in some cases, the holomorphic domain of $M\left(\tau, \tau^{-1}\left(\Lambda_{1}+\right.\right.$ $\left.\left.\rho_{O}^{Q}\right)\right)$ is extendable to the right side of the imaginary axis $\sqrt{-1} \operatorname{Re} \mathfrak{a}_{Q}$, however we do not know in general the asymptotic behavior of $f_{\tau}$ as $\left|\Im \Lambda_{1}\right| \rightarrow \infty$ in this region.

\section{$\S 7$. Examples}

ExAmple 1 . Let $V$ be an $n$-dimensional vector space defined over $k$, $G$ a group of linear automorphisms of $V$ and $\pi: G \rightarrow G$ the natural representation. We fix a free $\mathfrak{O}$-lattice $L$ in $V(k)$ and its $\mathfrak{D}$-basis $\mathbf{e}_{1}, \ldots, \mathbf{e}_{n}$. Then $V(k)$ and $G$ are identified with the column vector space $k^{n}$ and the general linear group $G L_{n}$, respectively. Let $P$ be the subgroup of upper triangular matrices and $Q$ the stabilizer in $G$ of the line spanned by $\mathbf{e}_{1}$. Then the map $g \mapsto \mathbf{e}_{1} \cdot g=g^{-1} \mathbf{e}_{1}$ yields an isomorphism from $X_{Q}=Q \backslash G$ to the projective space $\mathbb{P} V=\mathbb{P}^{n-1}$. Let $H_{\pi}$ be a height on $X_{Q}(k)$ defined as in Section 2. We take a maximal compact subgroup $K=\prod_{v \in \mathfrak{V}} K_{v}$ as follows:

$$
K_{v}= \begin{cases}G L_{n}\left(\mathfrak{O}_{v}\right) & \left(v \in \mathfrak{V}_{f}\right) \\ O(n) & (v \text { is a real place }) \\ U(n) & (v \text { is an imaginary place })\end{cases}
$$


For each $v \in \mathfrak{V}_{f}, \mathfrak{p}_{v}$ and $\mathfrak{f}_{v}$ stand for the maximal ideal of $\mathfrak{O}_{v}$ and the residual field $\mathfrak{O}_{v} / \mathfrak{p}_{v}$, respectively. If we set

$$
D_{v}=\left\{g \in K_{v}: g \equiv\left(\begin{array}{cc}
* & * \\
0 & \\
\vdots & * \\
0 &
\end{array}\right) \bmod \mathfrak{p}_{v}\right\},
$$

then $D_{v} \backslash K_{v}$ is isomorphic to $\mathbb{P}^{n-1}\left(\mathfrak{f}_{v}\right)$ by the reduction homomorphism. For every $x \in \mathbb{P}^{n-1}\left(k_{v}\right)$, there is an $h_{x} \in K_{v}$ such that $x=k_{v}\left(\mathbf{e}_{1} \cdot h_{x}\right)$. We denote by $[x]_{v}$ the reduction of $x$ modulo $\mathfrak{p}_{v}$, i.e., $[x]_{v}=\mathfrak{f}_{v}\left(\mathbf{e}_{1} \cdot h_{x} \bmod \mathfrak{p}_{v}\right)$. Let $\mathfrak{S}$ be a finite subset of $\mathfrak{V}_{f}$. We fix a point $\left(a_{v}\right)_{v \in \mathfrak{S}}$ in $\prod_{v \in \mathfrak{S}} \mathbb{P}^{n-1}\left(k_{v}\right)$ and set

$$
\begin{aligned}
& N\left(\mathbb{P}^{n-1}(k), T,\left(a_{v}\right)_{v \in \mathfrak{S})}\right. \\
& \quad=\mid\left\{x \in \mathbb{P}^{n-1}(k): H_{\pi}(x) \leq T \text { and }[x]_{v}=\left[a_{v}\right]_{v} \text { for all } v \in \mathfrak{S}\right\} \mid .
\end{aligned}
$$

It is obvious that

$$
N\left(\mathbb{P}^{n-1}(k), T,\left(a_{v}\right)_{v \in \mathfrak{S}}\right)=\left|E_{\pi}(D, T) \cdot h \cap X(k)\right|,
$$

where $D=K_{\infty} \times \prod_{v \in \mathfrak{S}} D_{v} \times \prod_{v \in \mathfrak{V}_{f}-\mathfrak{S}} K_{v}$ and $h=\left(h_{a_{v}}\right)_{v \in \mathfrak{S}} \times(e)_{v \in \mathfrak{V}-\mathfrak{S}} \in$ $K$. By Theorem 1 and the calculation of [W, Example 2], we have

$$
N\left(\mathbb{P}^{n-1}(k), T,\left(a_{v}\right)_{v \in \mathfrak{S}}\right) \sim \prod_{v \in \mathfrak{S}} \frac{\left|\mathfrak{f}_{v}\right|-1}{\left|\mathfrak{f}_{v}\right|^{n}-1} \cdot \frac{\operatorname{Res}_{s=1} \zeta_{k}(s)}{\left|D_{k}\right|^{(n-1) / 2} n Z_{k}(n)} \cdot T^{n[k: \mathbb{Q}]}
$$

as $T \rightarrow \infty$.

Here $\zeta_{k}(s)$ is the Dedekind zeta function of $k$,

$$
Z_{k}(s)=\left(\pi^{-s / 2} \Gamma(s / 2)\right)^{r_{1}}\left((2 \pi)^{1-s} \Gamma(s)\right)^{r_{2}} \zeta_{k}(s)
$$

and $r_{1}$ (resp. $r_{2}$ ) denotes a number of real (resp. imaginary) places of $k$. If $k=\mathbb{Q}$, this formula was proved in $[\mathrm{S}]$.

Example 2. Let $V, L$ and $\mathbf{e}_{1}, \ldots, \mathbf{e}_{n}$ be the same as in Example 1. Let $\Phi$ be a non-degenerate isotropic quadratic form on $V(k), G=S O_{\Phi}$ the special orthogonal group of $\Phi$ and $\pi: G \rightarrow G L(V)$ the natural representation. The height $H_{\pi}$ is the same as Example 1 . We assume $n \geq 4$ and $\Phi$ has the following matrix form with respect to the basis $\mathbf{e}_{1}, \ldots, \mathbf{e}_{n}$ :

$$
\Phi=\left(\begin{array}{lll} 
& & 1 \\
& \Phi_{0} & \\
1 & &
\end{array}\right)
$$


where $\Phi_{0}$ is a non-degenerate $(n-2) \times(n-2)$ symmetric matrix. Thus $\mathbf{e}_{1}$ is an isotropic vector of $\Phi$. Let $Q$ be the stabilizer in $G$ of the isotropic line spanned by $\mathbf{e}_{1}$. The map $g \mapsto \mathbf{e}_{1} \cdot g=g^{-1} \mathbf{e}_{1}$ gives rise to a $k$-rational embedding from $X_{\Phi}=Q \backslash G$ into $\mathbb{P}^{n-1}$. The image of $X_{\Phi}(k)$ is the set of all $\Phi$-isotropic lines $x \in \mathbb{P}^{n-1}(k)$. We put

$$
N\left(X_{\Phi}(k), T\right)=\left|\left\{x \in X_{\Phi}(k): H_{\pi}(x) \leq T\right\}\right| .
$$

Since the Levi-subgroup $M_{Q}$ is isomorphic to $G L_{1} \times S O_{\Phi_{0}}$, we have $\tau(G)=$ $\tau(Q)=2$ and $d_{G}=d_{Q}=1$, and furthermore, $e_{Q}=\operatorname{dim} U_{Q}=n-2$ and $e_{\pi}=1$. Therefore, Theorem 1 implies

$$
N\left(X_{\Phi}(k), T\right) \sim \frac{C_{G}}{(n-2) C_{Q}} T^{(n-2)[k: \mathbb{Q}]} \quad \text { as } T \rightarrow \infty .
$$

Here we supposed that $H_{\pi}$ is invariant by a good maximal compact subgroup $K$ of $G(\mathbb{A})$. The formula due to Ikeda [I, Theorems 9.6 and 9.7] deduces an explicit value of $C_{G} / C_{Q}$ for some choice of $K$. In the following, we state this formula. Let $\mathfrak{V}_{\infty}^{\prime}$ be the set of all real places of $k$. For every $v \in \mathfrak{V}, \mathbb{H}\left(k_{v}\right)$ denotes the hyperbolic plane $k_{v}^{2}$ endowed with the quadratic form $\left(\begin{array}{ll}0 & 1 \\ 1 & 0\end{array}\right)$. Then $V\left(k_{v}\right)$ is decomposed into the following form on $k_{v}$ :

$$
V\left(k_{v}\right)=\mathbb{H}\left(k_{v}\right)^{m_{v}} \oplus V_{v}^{0},
$$

where $V_{v}^{0}$ is a $\Phi$-anisotropic subspace. We put $\ell_{v}=\operatorname{dim} V_{v}^{0}$. In other words, $\left(n-\ell_{v}\right) / 2$ is the Witt index of $\Phi$ on $V\left(k_{v}\right)$. If $v \in \mathfrak{V}_{f}$, then $\ell_{v}$ is at most 4 . If $v \in \mathfrak{V}_{f}$ and $\ell_{v}=3$, then $V_{v}^{0}$ is identified with the space of pure quaternions of the division quaternion algebra $\mathbb{D}_{v}$ over $k_{v}$.

First, let $n$ be odd. We may assume without loss of generality that $\operatorname{det} \Phi_{0} \equiv 2(-1)^{(n-3) / 2}$ module $\left(k^{\times}\right)^{2}\left(\left[\mathrm{I}\right.\right.$, p. 207]). For every $v \in \mathfrak{V}_{f}$ with $\ell_{v}=3$, we take a maximal compact subgroup $K_{v}$ as the stabilizer in $G\left(k_{v}\right)$ of the lattice $\mathbb{H}\left(\mathfrak{O}_{v}\right)^{(n-3) / 2} \oplus\left(\mathfrak{O}_{\mathbb{D}_{v}} \cap V_{v}^{0}\right)$. Here $\mathfrak{O}_{\mathbb{D}_{v}}$ denotes the maximal order of $\mathbb{D}_{v}$. In other places $v$, we take $K_{v}$ as in [I, pp. 209-210]. Then

$$
\begin{aligned}
\frac{C_{G}}{C_{Q}}=\frac{\operatorname{Res}_{s=1} \zeta_{k}(s)}{\left|D_{k}\right|^{(n-2) / 2} Z_{k}(n-1)} \prod_{\substack{v \in \mathfrak{V}_{f} \\
\ell_{v}=3}} \frac{1-\left|\mathfrak{f}_{v}\right|^{-n+3}}{\left|\mathfrak{f}_{v}\right|\left(1-\left|\mathfrak{f}_{v}\right|^{-n+1}\right)} \\
\quad \times \prod_{v \in \mathfrak{V}_{\infty}^{\prime}} \prod_{i=1}^{\left[\left(\ell_{v}-1\right) / 4\right]} \frac{n-\ell_{v}+4 i-2}{n+\ell_{v}-4 i-2} .
\end{aligned}
$$


Next, let $n$ be even. We take a maximal compact subgroup $K_{v}$ as in [I, pp. 209-210] for every $v \in \mathfrak{V}$. Let $k^{\prime}=k\left(\sqrt{(-1)^{n / 2} \operatorname{det} \Phi}\right)$ be an extension of degree at most 2 over $k$ and let $\mathfrak{V}_{f}^{\prime}$ (resp. $\mathfrak{V}_{f}^{\prime \prime}$ ) be the set of $v \in \mathfrak{V}_{f}$ such that $\ell_{v}=2\left(\right.$ resp. $\left.\ell_{v}=4\right), v$ is unramified (resp. split) over $k^{\prime} / k$ and $\left.\Phi\right|_{V_{v}^{0}}$ is equivalent to the form $2 \varpi_{v} \cdot \operatorname{Norm}_{k_{v}^{\prime} / k_{v}}$, where $\varpi_{v}$ is a prime element of $k_{v}$ and $\operatorname{Norm}_{k_{v}^{\prime} / k_{v}}$ the norm form of the unramified quadratic extension $k_{v}^{\prime} / k_{v}$. Then

$$
\begin{aligned}
& \frac{C_{G}}{C_{Q}}=\frac{1}{\left|\mathfrak{f}_{\chi_{\Phi}}\right|^{1 / 2}\left|D_{k}\right|^{(n-2) / 2}} \frac{\operatorname{Res}_{s=1} \zeta_{k}(s)}{Z_{k}(n-2)} \frac{L\left(-1+n / 2, \chi_{\Phi}\right)}{L\left(n / 2, \chi_{\Phi}\right)} \\
& \quad \times \prod_{v \in \mathfrak{V}_{f}^{\prime}}\left|\mathfrak{f}_{v}\right|^{1-n / 2} \prod_{v \in \mathfrak{V}_{f}^{\prime \prime}} \frac{1-\left|\mathfrak{f}_{v}\right|^{2-n / 2}}{\left|\mathfrak{f}_{v}\right|\left(1-\left|\mathfrak{f}_{v}\right|^{-n / 2}\right)} \\
& \times \prod_{\substack{v \in \mathfrak{V}_{\infty}^{\prime} \\
\ell_{v} \equiv 0(4)}} \prod_{i=1}^{\ell_{v} / 4} \frac{n-4 i}{n+4 i-4} \prod_{\substack{v \in \mathfrak{V}_{\infty}^{\prime} \\
\ell_{v} \equiv 2(4)}} \prod_{i=1}^{\left(\ell_{v}-2\right) / 4} \frac{n-4 i-2}{n+4 i-2} .
\end{aligned}
$$

Here $\chi_{\Phi}$ is the quadratic character of $\mathbb{A}^{\times}$associated with $\Phi$, i.e.,

$$
\chi_{\Phi}(a)=\left\langle(-1)^{n / 2} \operatorname{det} \Phi, a\right\rangle
$$

for $a \in \mathbb{A}^{\times}$, where $\langle\cdot, \cdot\rangle$ is the Hilbert symbol, and $\mathfrak{f}_{\chi_{\Phi}}$ denotes the conductor of $\chi_{\Phi}$ and $L\left(s, \chi_{\Phi}\right)$ the Hecke $L$-function of $\chi_{\Phi}$.

\section{REFERENCES}

[B] A. Borel, Linear Algebraic Groups, Springer Verlag, 1991.

[BW] A. Borel and N. Wallach, Continuous Cohomology, Discrete Subgroups, and Representations of Reductive Groups, Princeton Univ. Press, 1980.

[BR] M. Borovoi and Z. Rudnick, Hardy-Littlewood varieties and semisimple groups, Invent. Math., 119 (1995), 37-66.

[DRS] W. Duke, Z. Rudnick and P. Sarnak, Density of integer points on affine homogeneous varieties, Duke Math. J., 71 (1993), 143-179.

[FMT] J. Franke, Y. I. Manin and Y. Tschinkel, Rational points of bounded height on Fano varieties, Invent. Math., 95 (1989), 421-435.

[G] R. Godement, Domaines fondamentaux des groupes arithmétiques, Exp. 257, Sém. Bourbaki, 15 (1962/1963).

[H] Harish-Chandra, Automorphic Forms on Semisimple Lie Groups, Lec. Notes in Math. 62, Springer Verlag, 1986.

[I] T. Ikeda, On the residue of the Eisenstein series and the Siegel-Weil formula, Comp. Mathematics., 103 (1996), 183-218. 
[Lai] K. F. Lai, Tamagawa number of reductive algebraic groups, Comp. Math., 47 (1980), 153-188.

[L] R. P. Langlands, The volume of the fundamental domain for some arithmetical subgroups of Chevalley groups, Proc. Symp. Pure Math. 9, Amer. Math. Soc. (1966), 143-148.

[MW] C. Moeglin and J.-L. Waldspurger, Spectral Decomposition and Eisenstein Series, Cambridge Univ. Press, 1995.

[MW1] M. Morishita and T. Watanabe, On S-Hardy-Littlewood homogeneous spaces, Int. J. Math., 9 (1998), 723-757.

[MW2] — Adele geometry of numbers, Class Field Theory - Its Centenary and Prospect, Adv. Studies in Pure Math. 30, Japan Math. Soc. (2001), pp. 509-536.

[S] A. Sato, Counting rational points on projective space with certain congruent conditions, preprint.

[W] T. Watanabe, On an analog of Hermite's constant, J. Lie Theory, 10 (2000), 33-52.

Department of Mathematics

Graduate School of Science

Osaka University

Toyonaka

Osaka, 560-0043

Japan

watanabe@math.wani.osaka-u.ac.jp 